This is an electronic reprint of the original article. This reprint may differ from the original in pagination and typographic detail.

Author(s): Tarpanov, D.; Toivanen, Jussi; Dobaczewski, Jacek; Carlsson, B. G.

Title: $\quad$ Polarization corrections to single-particle energies studied within the energy-densityfunctional and quasiparticle random-phase approximation approaches

Year: $\quad 2014$

Version:

Please cite the original version:

Tarpanov, D., Toivanen, J., Dobaczewski, J., \& Carlsson, B. G. (2014). Polarization corrections to single-particle energies studied within the energy-density-functional and quasiparticle random-phase approximation approaches. Physical Review C, 89(1), Article 014307. https://doi.org/10.1103/PhysRevC.89.014307

All material supplied via JYX is protected by copyright and other intellectual property rights, and duplication or sale of all or part of any of the repository collections is not permitted, except that material may be duplicated by you for your research use or educational purposes in electronic or print form. You must obtain permission for any other use. Electronic or print copies may not be offered, whether for sale or otherwise to anyone who is not an authorised user. 


\title{
Polarization corrections to single-particle energies studied within the energy-density-functional and quasiparticle random-phase approximation approaches
}

\author{
D. Tarpanov, ${ }^{1,2}$ J. Toivanen, ${ }^{3}$ J. Dobaczewski, ${ }^{1,3}$ and B. G. Carlsson ${ }^{4}$ \\ ${ }^{1}$ Institute of Theoretical Physics, Faculty of Physics, University of Warsaw, Hoża 69, PL-00-681 Warsaw, Poland \\ ${ }^{2}$ Institute for Nuclear Research and Nuclear Energy, 1784 Sofia, Bulgaria \\ ${ }^{3}$ Department of Physics, P.O. Box 35 (YFL), University of Jyväskylä, FI-40014 Jyväskylä, Finland \\ ${ }^{4}$ Division of Mathematical Physics, LTH, Lund University, Post Office Box 118, S-22100 Lund, Sweden
}

(Received 1 October 2013; published 13 January 2014)

\begin{abstract}
Background: Models based on using perturbative polarization corrections and mean-field blocking approximation give conflicting results for masses of odd nuclei.

Purpose: We systematically investigate the polarization and mean-field models, implemented within selfconsistent approaches that use identical interactions and model spaces, to find reasons for the conflicts between them.

Methods: For density-dependent interactions and with pairing correlations included, we derive and study links between the mean-field and polarization results obtained for energies of odd nuclei. We also identify and discuss differences between the polarization-correction and full particle-vibration-coupling (PVC) models. Numerical calculations are performed for the mean-field ground-state properties of deformed odd nuclei and then compared to the polarization corrections determined using the approach that conserves spherical symmetry.

Results: We have identified and numerically evaluated self-interaction (SI) energies that are at the origin of different results obtained within the mean-field and polarization-correction approaches.

Conclusions: Mean-field energies of odd nuclei are polluted by the SI energies, and this makes them different from those obtained using polarization-correction methods. A comparison of both approaches allows for the identification and determination of the SI terms, which then can be calculated and removed from the mean-field results, giving the self-interaction-free energies. The simplest deformed mean-field approach that does not break parity symmetry is unable to reproduce full PVC effects.
\end{abstract}

DOI: 10.1103/PhysRevC.89.014307

PACS number(s): 21.10.Pc, 21.60.Jz

\section{INTRODUCTION}

The perturbative particle-vibration-coupling (PVC) model for odd-particle-number nuclei emerges naturally from the self-consistent Green's function theory [1]. It describes the polarization of the nucleus when one particle is added or removed [2], and its results can, in principle, be directly compared against experimental data. As used in nuclear physics, the perturbative PVC method employs one-particle or one-hole states (or one-quasiparticle states) coupled with the random-phase approximation (RPA) or quasiparticle randomphase approximation (QRPA) excitations of an even-even reference nucleus and the residual nucleon-nucleon interaction that mixes these states at second-order perturbation theory. Numerous PVC calculations of increasing level of sophistication have already been performed; see, e.g., Refs. [3-19] and excellent recent reviews thereof available in Refs. [14,18,20].

An alternative to describing odd nuclei within the perturbative PVC calculations are the energy-density-functional (EDF) methods; see, e.g., Refs. [21-27], which use blocking of single-particle (s.p.) or quasiparticle orbitals. To distinguish these methods from the full PVC approach, in the present study we call them mean-field polarizations or polarization corrections. The advantage of blocked mean-field calculations is that they are nonperturbative and variational.

As it turns out, the effects obtained within the blocked mean-field methods are substantially different and, in general, weaker than those obtained from the perturbative PVC [20]. This discrepancy between models, even when using exactly the same interactions and model spaces, needs to be solved, and this is the main purpose of the present work.

The link between the mean-field and perturbative methods was proposed a long time ago [28-31]. Here we identify several approximations that are required to firmly establish such a link, and we also extend the derivations to EDFs based on density-dependent interactions and to those that include pairing correlations. Because the determination of mean-field polarizations requires breaking symmetries, no numerical comparison of the two approaches, such as given here, is available up to now. As required by a thorough comparison, both in the mean-field and (Q)RPA calculations we use full self-consistency and exactly the same particle-hole EDFs, pairing interactions, and model spaces.

The paper is built around two main chapters presenting theoretical derivations in Sec. II and the Appendix and numerical results in Sec. III. For theory, we present results pertaining to the Hartree-Fock (HF) approximation (Sec. II A), density functionals (Sec. IIB), and pairing correlations treated within the Hartree-Fock-Bogoliubov (HFB) framework (Sec. IIC). Then results of calculations are discussed for the density-independent (Sec. III A) and density-dependent (Sec. III B) particle-hole interactions and for paired systems (Sec. III C). Conclusions are given in Sec. IV. 


\section{THEORY}

\section{A. Polarization corrections in the HF approximation}

In this section we revisit the classic problem [28-31] of the polarization effect exerted by an odd particle on a mean-field state. To put further discussion in perspective, we study the problem in the HF approximation, and we assume that the mean field is obtained by the HF averaging [32] of a given known two-body density-independent interaction that has antisymmetrized matrix elements $\bar{v}_{i^{\prime} k^{\prime} i k}$.

Let $\rho^{A}$ and $h^{A}$ denote, respectively, the self-consistent density matrix and HF Hamiltonian for a system of $A$ fermions: $\operatorname{Tr} \rho^{A}=\mathrm{A},\left[h^{A}, \rho^{A}\right]=0$. Similarly, let $\rho^{A \pm 1}$ denote the self-consistent density matrices corresponding to the HF solution for the $(A \pm 1)$-particle system: $\operatorname{Tr} \rho^{A \pm 1}=A \pm 1$, $\left[h^{A \pm 1}, \rho^{A \pm 1}\right]=0$. We use notation in which the upper and lower signs correspond to adding or subtracting a particle. Without pairing correlations, even and odd systems are described in exactly the same way, so without any loss of generality, we assume that $A$ is even.

Self-consistent HF total energies of the $A$ - and $(A \pm 1)$ particle systems are given by [32]

$$
\begin{aligned}
E^{A} & =\operatorname{Tr}\left(t \rho^{A}\right)+\frac{1}{2} \operatorname{Tr}_{1} \operatorname{Tr}_{2}\left(\rho^{A} \bar{v} \rho^{A}\right), \\
E^{A \pm 1} & =\operatorname{Tr}\left(t \rho^{A \pm 1}\right)+\frac{1}{2} \operatorname{Tr}_{1} \operatorname{Tr}_{2}\left(\rho^{A \pm 1} \bar{v} \rho^{A \pm 1}\right) .
\end{aligned}
$$

Here $t$ represents the matrix of one-body kinetic energy. In what follows we always neglect the so-called center-of-mass correction to the kinetic energy [32,33]. These corrections are explicitly $A$-dependent and thus give trivial so-called mass polarization corrections [25] to energy differences $E^{A \pm 1}-$ $E^{A}$. Although they can always be added, they would obscure the analysis of standard polarization corrections, which are attributable to two-body interactions and which are the main focus of the present study.

Suppose now that $\rho^{\lambda}\left(\operatorname{Tr} \rho^{\lambda}=1\right)$ is the density matrix of a s.p. state $\lambda$. We may now ask what the relations are between the density matrices $\rho^{A}, \rho^{A \pm 1}$, and $\rho^{\lambda}$. Of course, we can always define a corrective density matrix $\delta \rho(\operatorname{Tr} \delta \rho=0)$ such that, by definition,

$$
\rho^{A \pm 1}=\rho^{A} \pm \rho^{\lambda}+\delta \rho .
$$

However, a perturbative treatment can only be obtained in the case when $\delta \rho$ is small, that is, small in the sense that when the energy of the odd systems $E^{A \pm 1}$ [Eq. (1b)] is calculated for the density matrix in Eq. (2), only terms up to second order in $\delta \rho$ are important.

Note that, by definition, the three density matrices are Hermitian and projective,

$$
\begin{aligned}
\left(\rho^{A}\right)^{2} & =\rho^{A}=\left(\rho^{A}\right)^{+}, \\
\left(\rho^{A \pm 1}\right)^{2} & =\rho^{A \pm 1}=\left(\rho^{A \pm 1}\right)^{+}, \\
\left(\rho^{\lambda}\right)^{2} & =\rho^{\lambda}=\left(\rho^{\lambda}\right)^{+} .
\end{aligned}
$$

Also note that $\delta \rho$ does depend on the polarizing state $\lambda$; nevertheless, we do not mark it with superscript $\lambda$. This is to avoid a confusion of understanding $\delta \rho$ as a correction to the orbital itself; indeed, this correction certainly corresponds to a modification of all orbitals of the system.

\section{Properties of $\delta \rho$}

The corrective density matrix $\delta \rho$ can be small only when the orbital $\lambda$ and the states in even and odd nuclei are chosen in a specific way. We may then have four interesting cases to consider. In the first case, let us assume that we initially solve the self-consistent equations of the even $A$-particle system and $|\lambda\rangle$ is one of the unoccupied HF eigenstates therein (a particle state); that is, $h^{A}|\lambda\rangle=e_{\lambda}|\lambda\rangle, \rho^{A} \rho^{\lambda}=0$. We may now put a particle in this orbital and solve the self-consistent equations of the $(A+1)$-particle system. In this sense, the $(A+1)$-particle system becomes polarized by an addition of a particle to the $A$-particle system. Note that by this procedure all HF s.p. states of the $A$-particle system become modified, including the added state $\lambda$.

The second case is obtained by a similar procedure, where instead we arrive at a polarized $(A-1)$-particle system. For that, we pick $\lambda$ as one of the occupied HF eigenstates of the $A$ particle system (a hole state); that is, $h^{A}|\lambda\rangle=e_{\lambda}|\lambda\rangle, \rho^{A} \rho^{\lambda}=$ $\rho^{\lambda}$. By removing a particle from this state and solving the self-consistent equations of the $(A-1)$-particle system, we now obtain the polarization correction corresponding to a hole. Note that for the two choices discussed up to now, the density matrices $\rho^{A}+\rho^{\lambda}$ and $\rho^{A}-\rho^{\lambda}$, are projective; that is,

$$
\left(\rho^{A} \pm \rho^{\lambda}\right)^{2}=\rho^{A} \pm \rho^{\lambda} \text {. }
$$

The two remaining interesting cases correspond to inverse polarizations, namely, we may initially solve the selfconsistent equations of the $(A \pm 1)$-particle systems, and then pick $\lambda$ either as an unoccupied orbital in the $(A-1)$ particle system, $h^{A-1}|\lambda\rangle=e_{\lambda}|\lambda\rangle, \rho^{A-1} \rho^{\lambda}=0$ or as an occupied orbital in the $(A+1)$-particle system, $h^{A+1}|\lambda\rangle=e_{\lambda}|\lambda\rangle$, $\rho^{A+1} \rho^{\lambda}=\rho^{\lambda}$. Of course, in both cases, the self-consistent equations solved for the $A$-particle system give the same solutions as before. However, now state $\lambda$ corresponds to the $(A \pm 1)$-particle systems, and thus density matrices $\rho^{A-1}+\rho^{\lambda}$ and $\rho^{A+1}-\rho^{\lambda}$, are projective; that is,

$$
\left(\rho^{A \mp 1} \pm \rho^{\lambda}\right)^{2}=\rho^{A \mp 1} \pm \rho^{\lambda} .
$$

We see that equations we are going to derive for the corrective density $\delta \rho$ do depend on the choices made for the state $\lambda$. For the direct polarizations, that is, when $\lambda$ is a self-consistent state in the even system, we square both sides of Eq. (2), and from Eq. (4) we obtain

$$
\delta \rho=\left(\rho^{A} \pm \rho^{\lambda}\right) \delta \rho+\delta \rho\left(\rho^{A} \pm \rho^{\lambda}\right)+(\delta \rho)^{2} .
$$

For the inverse polarizations, that is, when $\lambda$ is a self-consistent orbital in the odd systems, we rewrite Eq. (2) in the form

$$
\rho^{A \pm 1} \mp \rho^{\lambda}=\rho^{A}+\delta \rho
$$

and then square both sides, which from Eq. (5) gives

$$
\delta \rho=\rho^{A} \delta \rho+\delta \rho \rho^{A}+(\delta \rho)^{2} .
$$

Equations (6) and (8) allow us to derive specific properties of $\delta \rho$ that, however, are different for direct and inverse polarizations. Assuming that we can split $\delta \rho$ into terms of 
first, second, and higher (neglected) orders, that is,

$$
\delta \rho=\delta \rho^{(1)}+\delta \rho^{(2)}+\cdots,
$$

we now separately discuss direct and inverse polarizations. In what follows, we refer to the expansion in Eq. (9) as small-amplitude expansion, and we strive to discuss what an acceptable magnitude of $\rho^{\lambda}$ is, for which such an expansion is meaningful. As is well known, and as we explicitly show below, by keeping the lowest-order terms of the smallamplitude expansion one obtains the standard RPA equations.

Beginning with the inverse polarizations, Eq. (8) gives

$$
\begin{aligned}
& \delta \rho^{(1)}=\rho^{A} \delta \rho^{(1)}+\delta \rho^{(1)} \rho^{A}, \\
& \delta \rho^{(2)}=\rho^{A} \delta \rho^{(2)}+\delta \rho^{(2)} \rho^{A}+\left(\delta \rho^{(1)}\right)^{2} .
\end{aligned}
$$

We can now discuss properties of the standard particle-hole (ph), particle-particle (pp), and hole-hole (hh) matrix elements of $\delta \rho$, where the particle and hole states correspond to the unoccupied and occupied states, respectively, in the even Aparticle system; that is,

$$
\rho_{\mathrm{hh}^{\prime}}^{A}=\delta_{\mathrm{hh}^{\prime}}, \quad \rho_{\mathrm{ph}}^{A}=0, \quad \rho_{\mathrm{hp}}^{A}=0, \quad \rho_{\mathrm{pp}^{\prime}}^{A}=0 .
$$

We note here that the notion of particle and hole states always pertains to a specific projective density matrix. By definition, the particle and hole states are the eigenstates of the density matrix with eigenvalues 0 and 1 , respectively. In the even and time-even system, both particle and hole states are doubly Kramers degenerate and for each such a pair, both degenerate partner states are simultaneously either occupied or empty. This creates the standard image of the Fermi "sphere," whereby the pairs of states below or above the Fermi level are occupied or empty, respectively. This image is particularly conspicuous in semimagic spherical systems, wherein the entire $(2 j+1)$ degenerate orbitals are either occupied or empty. We described here this standard image in such detail because below we contrast it with a nonstandard representation of particle-hole states pertaining to the odd system.

Equation (10a) does not put any constraint on the ph matrix elements of $\delta \rho^{(1)}$, and it requires that its pp and hh matrix elements vanish identically. Therefore, the leadingorder (second-order) $\mathrm{pp}$ and hh matrix elements of $\delta \rho$ are determined by Eq. (10b), and they solely depend on the leading-order (first-order) ph matrix elements thereof; that is,

$$
\begin{aligned}
& \delta \rho_{\mathrm{pp}^{\prime}}^{(2)}=\sum_{\mathrm{h}} \delta \rho_{\mathrm{ph}}^{(1)} \delta \rho_{\mathrm{hp}^{\prime}}^{(1)}, \\
& \delta \rho_{\mathrm{hh}^{\prime}}^{(2)}=-\sum_{\mathrm{p}} \delta \rho_{\mathrm{hp}}^{(1)} \delta \rho_{\mathrm{ph}^{\prime}}^{(1)} .
\end{aligned}
$$

We see that the standard ph structure of $\delta \rho$, pertaining to the A-particle system, appears for the inverse polarizations. However, as we derived above, in this case the polarizing state $\lambda$ must be calculated in the odd system.

Let us next discuss the direct polarizations, for which Eq. (6) holds. The small-amplitude expansion (9) then gives

$$
\begin{aligned}
& \delta \rho^{(1)}=\left(\rho^{A} \pm \rho^{\lambda}\right) \delta \rho^{(1)}+\delta \rho^{(1)}\left(\rho^{A} \pm \rho^{\lambda}\right), \\
& \delta \rho^{(2)}=\left(\rho^{A} \pm \rho^{\lambda}\right) \delta \rho^{(2)}+\delta \rho^{(2)}\left(\rho^{A} \pm \rho^{\lambda}\right)+\left(\delta \rho^{(1)}\right)^{2} .
\end{aligned}
$$

Equations (13) are similar to those in Eq. (10), with the notable difference of the odd-system density matrix $\rho^{A} \pm \rho^{\lambda}$ replacing the even-system density matrix $\rho^{A}$. This is important, because the analysis of the ph structure of $\delta \rho^{(1)}$ must now pertain to the eigenvalues of $\rho^{A} \pm \rho^{\lambda}$ and not to those of $\rho^{A}$.

We see that the hole and particle states, which now are denoted by symbols $\mathrm{H}$ and $\mathrm{P}$, respectively, are identical to the previous hole and particle states, $\mathrm{h}$ and $\mathrm{p}$; however, the polarizing state $\lambda$ is an exception. If we consider the $(A+1)$ particle system, the state $\lambda$, which previously was a particle state $\mathrm{p}=\lambda$, is now an eigenstate of density matrix $\rho^{A}+\rho^{\lambda}$ with eigenvalue 1 ; that is, it is a hole state $\mathrm{H}=\lambda$. Similarly, for an $(A-1)$-particle system, the previous hole state $\mathrm{h}=\lambda$ is now an eigenstate of density matrix $\rho^{A}-\rho^{\lambda}$ with eigenvalue 0 ; that is, it is a particle state $\mathrm{P}=\lambda$. We should also remember, that the Kramers-degenerate partner $\bar{\lambda}$ of the polarizing state $\lambda$ now stays on the opposite side of the Fermi surface; namely, when $\lambda$ is a hole state, $\bar{\lambda}$ is a particle state and vice versa. As we see, a nonstandard form of the Fermi "sphere" must now be considered.

Altogether, the hole $(\mathrm{H})$ and particle $(\mathrm{P})$ states are defined by

$$
\begin{gathered}
\left(\rho^{A} \pm \rho^{\lambda}\right)_{\mathrm{HH}^{\prime}}=\delta_{\mathrm{HH}^{\prime}}, \quad\left(\rho^{A} \pm \rho^{\lambda}\right)_{\mathrm{PH}}=0, \\
\left(\rho^{A} \pm \rho^{\lambda}\right)_{\mathrm{HP}}=0, \quad\left(\rho^{A} \pm \rho^{\lambda}\right)_{\mathrm{PP}^{\prime}}=0 .
\end{gathered}
$$

Then matrix elements $\delta \rho_{\mathrm{PH}}^{(1)}$ are unconstrained, whereas the PP and $\mathrm{HH}$ matrix elements of the second-order correction are given by

$$
\begin{gathered}
\delta \rho_{\mathrm{PP}^{\prime}}^{(2)}=\sum_{\mathrm{H}} \delta \rho_{\mathrm{PH}}^{(1)} \delta \rho_{\mathrm{HP}^{\prime}}^{(1)}, \\
\delta \rho_{\mathrm{HH}^{\prime}}^{(2)}=-\sum_{\mathrm{P}} \delta \rho_{\mathrm{HP}}^{(1)} \delta \rho_{\mathrm{PH}^{\prime}}^{(1)} .
\end{gathered}
$$

It is evident that the nonstandard structure of the first-order density matrix $\delta \rho^{(1)}$ implies solving the RPA equations directly in the odd system and thus precludes using the spherical and time-reversal symmetries.

In summary, for direct polarizations, for which the polarizing state $\lambda$ is calculated in the even system, we obtain the nonstandard PH structure (15) of $\delta \rho$. However, for inverse polarizations, for which the polarizing state $\lambda$ is calculated in the odd system, we obtain the standard ph structure (12) of $\delta \rho$. From these considerations, it appears that a rigorous analysis of the HF polarization effects, which would be based on elements solely determined in the even system, does not exist, and one must make further simplifying assumptions. The easiest way out is to neglect the differences between the polarizing states calculated in the even and odd systems and to use equations pertaining to inverse polarizations along with the polarizing state $\lambda$ determined in the even system. In what follows, we use this strategy.

\section{Corrections to energies}

Equations for the polarization corrections to the s.p. energies can be derived by comparing the self-consistent 
energies in even and odd systems. Inserting the odd-system density matrices (2) into the odd-system energy (1b), we obtain

$$
\begin{aligned}
E^{A \pm 1}= & E^{A} \pm t_{\lambda \lambda}+\sum_{i i^{\prime}} t_{i^{\prime} i} \delta \rho_{i i^{\prime}}+\frac{1}{2} \bar{v}_{\lambda \lambda \lambda \lambda} \\
& +\frac{1}{2} \sum_{i i^{\prime} k k^{\prime}} \delta \rho_{i^{\prime} i} \bar{v}_{i k^{\prime} i^{\prime} k} \delta \rho_{k k^{\prime}} \pm \frac{1}{2} \sum_{i i^{\prime}} \rho_{i^{\prime} i}^{A} \bar{v}_{i \lambda i^{\prime} \lambda} \\
& \pm \frac{1}{2} \sum_{k k^{\prime}} \bar{v}_{\lambda k^{\prime} \lambda k} \rho_{k k^{\prime}}^{A} \pm \frac{1}{2} \sum_{i i^{\prime}} \delta \rho_{i^{\prime} i} \bar{v}_{i \lambda i^{\prime} \lambda} \\
& \pm \frac{1}{2} \sum_{k k^{\prime}} \bar{v}_{\lambda k^{\prime} \lambda k^{\prime}} \delta \rho_{k k^{\prime}}+\frac{1}{2} \sum_{i i^{\prime} k k^{\prime}} \rho_{i^{\prime} i}^{A} \bar{v}_{i k^{\prime} i^{\prime} k} \delta \rho_{k k^{\prime}} \\
& +\frac{1}{2} \sum_{i i^{\prime} k k^{\prime}} \delta \rho_{i^{\prime} i} \bar{v}_{i k^{\prime} i^{\prime} k} \rho_{k k^{\prime}}^{A}
\end{aligned}
$$

We now use the following facts and definitions:

$$
\begin{aligned}
h_{i^{\prime} i}^{A} & =t_{i^{\prime} i}+\sum_{k k^{\prime}} \bar{v}_{i^{\prime} k^{\prime} i k} \rho_{k k^{\prime}}^{A}, \\
e_{\lambda} & =h_{\lambda \lambda}^{A}, \\
0 & =\bar{v}_{\lambda \lambda \lambda \lambda}, \\
h_{i^{\prime} i}^{\lambda} & =\bar{v}_{i^{\prime} \lambda i \lambda}, \\
\delta h_{i^{\prime} i} & =\sum_{k k^{\prime}} \bar{v}_{i^{\prime} k^{\prime} i k} \delta \rho_{k k^{\prime}} .
\end{aligned}
$$

Equation (17a) is the standard definition of the HF mean field in the $A$-particle system and $e_{\lambda}(17 \mathrm{~b})$ is its diagonal matrix element in the self-consistent basis. Equation (17c) is a simple consequence of the antisymmetry of the two-body matrix elements and represents the fact that in the HF approximation there is no self-interaction (SI). Equations (17d) and (17e) define the mean-field potentials generated by the polarizing state $\lambda$ and correction $\delta \rho$, respectively. In terms of these definitions, the odd-system energy can be written as

$$
\begin{aligned}
E^{A \pm 1}= & E^{A} \pm e_{\lambda}+\sum_{i i^{\prime}} h_{i^{\prime} i}^{A} \delta \rho_{i i^{\prime}} \\
& \pm \sum_{i i^{\prime}} h_{i^{\prime} i}^{\lambda} \delta \rho_{i i^{\prime}}+\frac{1}{2} \sum_{i i^{\prime}} \delta h_{i^{\prime} i} \delta \rho_{i i^{\prime}} .
\end{aligned}
$$

Up to now, expression (18) is exact. To simplify it, we can use the small-amplitude expansion (9) and thus conditions (12), and neglect terms beyond second order. In the basis of particle and hole states, the mean-field Hamiltonian $h_{i^{\prime} i}^{A}$ is by definition diagonal; therefore, owing to Eqs. (12), the third term on the right-hand side is of the second order in $\delta \rho^{(1)}$. Similarly, the fifth term is obviously of the second order too. However, unless we assume that $h^{\lambda}$ is small (of the first order), the fourth term may contain subleading second-order terms, including the pp and hh matrix elements of $\delta \rho^{(2)}$, which do not appear in the standard RPA method. Therefore, to have a consistent RPA-type second-order expression for the energy of the $A \pm 1$ system, we must make the assumption of $h^{\lambda}$ being small as compared to $h^{A}$. This assumption can also be understood as $\rho^{\lambda}$ being small as compared to $\rho^{A}$, that is, the system being appropriately heavy.

In fact, such an assumption can partially be tested by keeping the leading-order (second-order) $\mathrm{pp}^{\prime}$ and $\mathrm{hh}^{\prime}$ matrix elements of the fourth term, which depend on the leadingorder (first-order) matrix elements of $\delta \rho$. Then we obtain the following approximate expression:

$$
\begin{aligned}
E^{A \pm 1}= & E^{A} \pm e_{\lambda}+\sum_{\mathrm{ph}}\left(e_{p}-e_{h}\right) \delta \rho_{\mathrm{ph}} \delta \rho_{\mathrm{hp}} \\
& +\frac{1}{2} \sum_{\mathrm{ph}} \delta h_{\mathrm{ph}} \delta \rho_{\mathrm{hp}}+\frac{1}{2} \sum_{\mathrm{ph}} \delta h_{\mathrm{hp}} \delta \rho_{\mathrm{ph}} \\
& \pm \sum_{\mathrm{pp} \mathrm{h}^{\prime}} h_{\mathrm{p}^{\prime} \mathrm{p}}^{\lambda} \delta \rho_{\mathrm{ph}} \delta \rho_{\mathrm{hp}^{\prime}} \mp \sum_{\mathrm{hh} \mathrm{p}^{\prime}} h_{\mathrm{h}^{\prime} \mathrm{h}}^{\lambda} \delta \rho_{\mathrm{hp}} \delta \rho_{\mathrm{ph}^{\prime}} \\
& \pm \sum_{\mathrm{ph}} h_{\mathrm{ph}}^{\lambda} \delta \rho_{\mathrm{hp}} \pm \sum_{\mathrm{ph}} h_{\mathrm{hp}}^{\lambda} \delta \rho_{\mathrm{ph}} .
\end{aligned}
$$

This can be summarized in the form of polarization corrections to energies of odd states $\delta E$,

$$
E^{A \pm 1}=E^{A} \pm e_{\lambda}+\delta E
$$

or polarization corrections to s.p. energies $\delta e_{\lambda}$,

$$
E^{A \pm 1}=E^{A} \pm\left(e_{\lambda}+\delta e_{\lambda}\right)
$$

for

$$
\begin{aligned}
\delta E= \pm \delta e_{\lambda} & =\frac{1}{2}\left(\begin{array}{ll}
\delta \rho^{*}, & \delta \rho
\end{array}\right)\left(\begin{array}{cc}
A^{\prime} & B \\
B^{*} & A^{\prime *}
\end{array}\right)\left(\begin{array}{c}
\delta \rho \\
\delta \rho^{*}
\end{array}\right) \\
& \pm\left(\begin{array}{ll}
\delta \rho^{*}, & \delta \rho
\end{array}\right)\left(\begin{array}{c}
h^{\lambda} \\
h^{\lambda *}
\end{array}\right),
\end{aligned}
$$

where $\delta \rho$ and $h^{\lambda}$ represent vectors of ph matrix elements, $\delta \rho_{\mathrm{ph}}$ and $h_{\mathrm{ph}}^{\lambda}$, respectively; that is,

$$
\begin{aligned}
& h_{\mathrm{ph}}^{\lambda}=\bar{v}_{\mathrm{p} \lambda \mathrm{h} \lambda}, \\
& h_{\mathrm{ph}}^{\lambda *}=h_{\mathrm{hp}}^{\lambda}=\bar{v}_{\mathrm{h} \lambda \mathrm{p} \lambda},
\end{aligned}
$$

and matrices $A^{\prime}$ and $B$,

$$
\begin{aligned}
& A_{\mathrm{p}^{\prime} \mathrm{h}^{\prime}, \mathrm{ph}}^{\prime}=A_{\mathrm{p}^{\prime} \mathrm{h}^{\prime}, \mathrm{ph}} \pm h_{\mathrm{p}^{\prime} \mathrm{p}}^{\lambda} \delta_{\mathrm{h}^{\prime} \mathrm{h}} \mp h_{\mathrm{hh}^{\prime}}^{\lambda} \delta_{\mathrm{pp}^{\prime}}, \\
& A_{\mathrm{p}^{\prime} \mathrm{h}^{\prime}, \mathrm{ph}}=\left(e_{\mathrm{p}}-e_{\mathrm{h}}\right) \delta_{\mathrm{pp}^{\prime}} \delta_{\mathrm{hh}^{\prime}}+\bar{v}_{\mathrm{hp}^{\prime} \mathrm{ph}^{\prime}}, \\
& B_{\mathrm{p}^{\prime} \mathrm{h}^{\prime}, \mathrm{ph}}=\bar{v}_{\mathrm{pp}^{\prime} \mathrm{hh}^{\prime}},
\end{aligned}
$$

build the RPA matrix $\left(\begin{array}{cc}A^{\prime} & B \\ B^{*} & A^{\prime *}\end{array}\right)$.

We see that the second-order terms depending on $h^{\lambda}$, which we have kept in Eq. (19), lead to modified matrix elements $A_{\mathrm{p}^{\prime} \mathrm{h}^{\prime}, \mathrm{ph}}^{\prime}$, as compared to the standard RPA matrix $A_{\mathrm{p}^{\prime} \mathrm{h}^{\prime}, \mathrm{ph}}$. In this formulation, the RPA equations do depend on the polarizing state $\lambda$. In Sec. III, we perform numerical calculations with and without these terms, and we check that they play a minor role and can be safely omitted, thus supporting the validity of the assumption about the smallness of $h^{\lambda}$.

\section{Equation for $\delta \rho$}

Equation for the correction $\delta \rho$ can be derived from the fact that the density matrix of Eq. (2) is a self-consistent solution 
of the HF equations in the ( $A \pm 1)$-particle system,

$$
0=\left[h^{A \pm 1}, \rho^{A \pm 1}\right]=\left[h^{A} \pm h^{\lambda}+\delta h, \rho^{A} \pm \rho^{\lambda}+\delta \rho\right] .
$$

As discussed above, we neglect differences between states $\lambda$ calculated in even and odd systems; that is, we have $\left[h^{A \pm 1}, \rho^{\lambda}\right]=0$. Moreover, because $\rho^{A}$ is the self-consistent solution of the $A$-particle system, we have $\left[h^{A}, \rho^{A}\right]=0$, which gives

$$
0=\left[h^{A}, \delta \rho\right] \pm\left[h^{\lambda}, \rho^{A}\right] \pm\left[h^{\lambda}, \delta \rho\right]+\left[\delta h, \rho^{A}\right]+[\delta h, \delta \rho] .
$$

In the leading (first) order, the last term, quadratic in the density $\delta \rho$, can be dropped, and we also drop the second-order matrix elements $\delta \rho_{\mathrm{pp}^{\prime}}$ and $\delta \rho_{\mathrm{hh}^{\prime}}$. Then, the ph and hp matrix elements of the above equation read

$$
\begin{aligned}
0 & =\left(e_{\mathrm{p}}-e_{\mathrm{h}}\right) \delta \rho_{\mathrm{ph}} \pm h_{\mathrm{ph}}^{\lambda} \pm \sum_{\mathrm{p}^{\prime}} h_{\mathrm{pp}^{\prime}}^{\lambda} \delta \rho_{\mathrm{p}^{\prime} \mathrm{h}} \mp \sum_{\mathrm{h}^{\prime}} \delta \rho_{\mathrm{ph}^{\prime}} h_{\mathrm{h}^{\prime} \mathrm{h}}^{\lambda}+\delta h_{\mathrm{ph}} \\
& =\left(A^{\prime} \delta \rho\right)_{\mathrm{ph}}+\left(B \delta \rho^{*}\right)_{\mathrm{ph}} \pm h_{\mathrm{ph}}^{\lambda}, \\
0 & =\left(e_{\mathrm{h}}-e_{\mathrm{p}}\right) \delta \rho_{\mathrm{hp}} \mp h_{\mathrm{hp}}^{\lambda} \pm \sum_{\mathrm{h}^{\prime}} h_{\mathrm{hh}^{\prime}}^{\lambda} \delta \rho_{\mathrm{h}^{\prime} \mathrm{p}} \mp \sum_{\mathrm{p}^{\prime}} \delta \rho_{\mathrm{h}^{\prime}} h_{\mathrm{p}^{\prime} \mathrm{p}}^{\lambda}-\delta h_{\mathrm{hp}} \\
& =-\left(A^{*} \delta \rho^{*}\right)_{\mathrm{ph}}-\left(B^{*} \delta \rho\right)_{\mathrm{ph}} \mp h_{\mathrm{ph}}^{\lambda *},
\end{aligned}
$$

and in the matrix notation they can be written as

$$
\left(\begin{array}{cc}
A^{\prime} & B \\
B^{*} & A^{\prime *}
\end{array}\right)\left(\begin{array}{c}
\delta \rho \\
\delta \rho^{*}
\end{array}\right)=\mp\left(\begin{array}{c}
h^{\lambda} \\
h^{\lambda *}
\end{array}\right) .
$$

Here again we see that the matrix elements of $h^{\lambda}$ must be at least of the same order (the first order) as are those of $\delta \rho$.

Condition (28) is exactly equal to the condition that the total energy of the odd system (20) is stationary with respect to correction $\delta \rho$. In other words, vanishing variation of $\delta E$, Eq. (22), with respect to $\delta \rho$ gives Eq. (28). Then, at the stationary point, the correction to the total energy reads

$$
\delta E=-\frac{1}{2}\left(\delta \rho^{*}, \quad \delta \rho\right)\left(\begin{array}{cc}
A^{\prime} & B \\
B^{*} & A^{*}
\end{array}\right)\left(\begin{array}{c}
\delta \rho \\
\delta \rho^{*}
\end{array}\right) ;
$$

that is, for a positive-definite RPA matrix, the correction to the total energy is always negative, irrespective of adding or subtracting a particle. For the corrections to s.p. energies we have

$$
\delta e_{\lambda}=\mp \frac{1}{2}\left(\delta \rho^{*}, \quad \delta \rho\right)\left(\begin{array}{cc}
A^{\prime} & B \\
B^{*} & A^{\prime *}
\end{array}\right)\left(\begin{array}{c}
\delta \rho \\
\delta \rho^{*}
\end{array}\right)
$$

that is, particle states move down and hole states move up. In view of Eq. (28), corrections (30) can also be written in two other equivalent forms:

$$
\begin{array}{ll}
\delta e_{\lambda}=\mp \frac{1}{2}\left(h^{\lambda *},\right. & \left.h^{\lambda}\right)\left(\begin{array}{cc}
A^{\prime} & B \\
B^{*} & A^{* *}
\end{array}\right)^{-1}\left(\begin{array}{c}
h^{\lambda} \\
h^{\lambda *}
\end{array}\right), \\
\delta e_{\lambda}=-\frac{1}{2}\left(\delta \rho^{*},\right. & \delta \rho)\left(\begin{array}{c}
h^{\lambda} \\
h^{\lambda *}
\end{array}\right) .
\end{array}
$$

In Eq. (31), the inverse of the RPA matrix can be calculated either through its eigenvectors or through the RPA amplitudes. In the second case, we use the RPA equations and completeness relations [32],

$$
\begin{aligned}
\left(\begin{array}{cc}
A^{\prime} & B \\
B^{*} & A^{\prime *}
\end{array}\right)\left(\begin{array}{cc}
X & -Y^{*} \\
Y & -X^{*}
\end{array}\right) & =\left(\begin{array}{cc}
X & Y^{*} \\
-Y & -X^{*}
\end{array}\right)\left(\begin{array}{cc}
\hbar \omega & 0 \\
0 & \hbar \omega
\end{array}\right), \\
\left(\begin{array}{cc}
X & -Y^{*} \\
Y & -X^{*}
\end{array}\right)\left(\begin{array}{ll}
X^{+} & -Y^{+} \\
Y^{T} & -X^{T}
\end{array}\right) & =\left(\begin{array}{cc}
1 & 0 \\
0 & 1
\end{array}\right),
\end{aligned}
$$

where $\hbar \omega$ is a diagonal matrix of positive RPA eigenvalues. This gives

$$
\left(\begin{array}{cc}
A^{\prime} & B \\
B^{*} & A^{\prime *}
\end{array}\right)=\left(\begin{array}{cc}
X & Y^{*} \\
-Y & -X^{*}
\end{array}\right)\left(\begin{array}{cc}
\hbar \omega & 0 \\
0 & \hbar \omega
\end{array}\right)\left(\begin{array}{cc}
X^{\dagger} & -Y^{\dagger} \\
Y^{T} & -X^{T}
\end{array}\right)
$$

The inverse of the RPA matrix exists if all eigenvalues are nonzero and has the form

$$
\begin{aligned}
& \left(\begin{array}{cc}
A^{\prime} & B \\
B^{*} & A^{\prime *}
\end{array}\right)^{-1} \\
& \quad=\left(\begin{array}{cc}
X & -Y^{*} \\
Y & -X^{*}
\end{array}\right)\left(\begin{array}{cc}
\hbar \omega^{-1} & 0 \\
0 & \hbar \omega^{-1}
\end{array}\right)\left(\begin{array}{cc}
X^{\dagger} & Y^{\dagger} \\
-Y^{T} & -X^{T}
\end{array}\right) .
\end{aligned}
$$

Finally, in terms of the RPA amplitudes and energies, corrections (31) then become equal to [29]

$$
\delta e_{\lambda}=\mp \sum_{\omega>0} \frac{\left|\sum_{\mathrm{ph}} h_{\mathrm{ph}}^{\lambda *} X_{\mathrm{ph}}^{\omega}+h_{\mathrm{ph}}^{\lambda} Y_{\mathrm{ph}}^{\omega}\right|^{2}}{\hbar \omega} .
$$

\section{B. Polarization corrections for density functionals}

Let us now rederive the polarization corrections of Sec. II A for the case of the total energy given by a minimization of an EDF and not of the HF average of a Hamiltonian. Differences between these two cases can be of dual origin. First, a quasilocal EDF built as the most general quadratic function of densities deviates from a HF average of a zerorange momentum-dependent interaction unless its coupling constants obey a specific set of linear conditions; see, e.g., Refs. [34,35]. For the Skyrme EDF, these conditions can be formulated as a linear dependence of the time-odd coupling constants on the time-even ones, and a linear dependence between the isovector and isoscalar spin-orbit coupling constants [36]. In this work we only consider EDFs of this type. The second reason for differences arises because of so-called density-dependent interactions, which also lead to EDFs that are not equal to HF averages of Hamiltonians.

Focusing on this second case, we now consider EDFs determined by the HF averaging of antisymmetrized densitydependent matrix elements $\bar{v}_{i^{\prime} k^{\prime} i k}[\rho]$. Then, the total energies read

$$
\begin{aligned}
E^{A} & =\operatorname{Tr}\left(t \rho^{A}\right)+\frac{1}{2} \operatorname{Tr}_{1} \operatorname{Tr}_{2}\left(\rho^{A} \bar{v}\left[\rho^{A}\right] \rho^{A}\right), \\
E^{A \pm 1} & =\operatorname{Tr}\left(t \rho^{A \pm 1}\right)+\frac{1}{2} \operatorname{Tr}_{1} \operatorname{Tr}_{2}\left(\rho^{A \pm 1} \bar{v}\left[\rho^{A \pm 1}\right] \rho^{A \pm 1}\right) .
\end{aligned}
$$

We see that both energies, apart from the standard quadratic dependencies on densities [cf. Eqs. (1)], do depend on densities through the density dependence of interactions. These latter dependencies preclude comparing energies of even and odd 
systems, unless we make the simplifying assumption that $\bar{v}\left[\rho^{A \pm 1}\right]$ and $\bar{v}\left[\rho^{A}\right]$ can be connected by a second-order expansion in $\rho^{A \pm 1}-\rho^{A}$. From Eq. (2) we see again that this requires $\rho^{\lambda}$ to be of the same (first) order as $\delta \rho$. Under this assumption, we have

$$
\begin{aligned}
& \bar{v}_{i^{\prime} k^{\prime} i k}\left[\rho^{A \pm 1}\right] \\
& \simeq \bar{v}_{i^{\prime} k^{\prime} i k}\left[\rho^{A}\right] \pm \sum_{m n} \frac{\partial \bar{v}_{i^{\prime} k^{\prime} i k}}{\partial \rho_{m n}}\left(\rho_{m n}^{\lambda} \pm \delta \rho_{m n}\right) \\
& \quad+\frac{1}{2} \sum_{m^{\prime} n^{\prime} m n} \frac{\partial^{2} \bar{v}_{i^{\prime} k^{\prime} i k}}{\partial \rho_{m n} \partial \rho_{m^{\prime} n^{\prime}}}\left(\rho_{m n}^{\lambda} \pm \delta \rho_{m n}\right)\left(\rho_{m^{\prime} n^{\prime}}^{\lambda} \pm \delta \rho_{m^{\prime} n^{\prime}}\right)
\end{aligned}
$$

where all partial derivatives must be evaluated at $\rho \equiv \rho^{A}$.

We can now insert Eqs. (2) and (39) into the odd-system energy (38b) and obtain up to the second order in $\pm \rho^{\lambda}+\delta \rho$,

$$
\begin{aligned}
E^{A \pm 1}= & E^{A}+\operatorname{Tr} \tilde{h}^{A}\left( \pm \rho^{\lambda}+\delta \rho\right) \\
& +\frac{1}{2} \operatorname{Tr}_{1} \operatorname{Tr}_{2}\left( \pm \rho^{\lambda}+\delta \rho\right) \tilde{\tilde{v}}\left( \pm \rho^{\lambda}+\delta \rho\right),
\end{aligned}
$$

where the mean-field Hamiltonian $\tilde{h}^{A}$,

$$
\tilde{h}_{i^{\prime} i}^{A}=t_{i^{\prime} i}+\sum_{k k^{\prime}} \tilde{v}_{i^{\prime} k^{\prime} i k} \rho_{k k^{\prime}}^{A},
$$

and effective two-body matrix elements, $\tilde{v}_{i^{\prime} k^{\prime} i k}$ and $\tilde{\tilde{v}}_{i^{\prime} k^{\prime} i k}$, contain rearrangement terms,

$$
\begin{aligned}
\tilde{v}_{i^{\prime} k^{\prime} i k}= & \bar{v}_{i^{\prime} k^{\prime} i k}+\frac{1}{2} \sum_{j^{\prime} j} \frac{\partial \bar{v}_{j^{\prime} k^{\prime} j k}}{\partial \rho_{i i^{\prime}}} \rho_{j j^{\prime}}^{A}, \\
\tilde{\tilde{v}}_{i^{\prime} k^{\prime} i k}= & \bar{v}_{i^{\prime} k^{\prime} i k}+\sum_{j^{\prime} j}\left(\frac{\partial \bar{v}_{j^{\prime} k^{\prime} j k}}{\partial \rho_{i i^{\prime}}}+\frac{\partial \bar{v}_{j^{\prime} i^{\prime} j i}}{\partial \rho_{k k^{\prime}}}\right) \rho_{j j^{\prime}}^{A} \\
& +\frac{1}{2} \sum_{j^{\prime} m^{\prime} j m} \frac{\partial^{2} \bar{v}_{j^{\prime} m^{\prime} j m}}{\partial \rho_{i i^{\prime}} \partial \rho_{k k^{\prime}}} \rho_{j j^{\prime}}^{A} \rho_{m m^{\prime}}^{A} .
\end{aligned}
$$

The redefined two-body matrix elements allow us to write the odd-system energy in the form analogous to Eq. (18),

$$
\begin{aligned}
E^{A \pm 1}= & E^{A} \pm e_{\lambda}+\sum_{i i^{\prime}} \tilde{h}_{i^{\prime} i}^{A} \delta \rho_{i i^{\prime}}+\frac{1}{2} \tilde{\tilde{h}}_{\lambda \lambda}^{\lambda} \\
& \pm \sum_{i i^{\prime}} \tilde{\tilde{h}}_{i^{\prime} i}^{\lambda} \delta \rho_{i i^{\prime}}+\frac{1}{2} \sum_{i i^{\prime}} \delta \tilde{\tilde{h}}_{i^{\prime} i} \delta \rho_{i i^{\prime}},
\end{aligned}
$$

but with the following redefinitions,

$$
\begin{aligned}
e_{\lambda} & =\tilde{h}_{\lambda \lambda}^{A} \\
\tilde{\tilde{h}}_{\lambda \lambda}^{\lambda} & =\tilde{\tilde{v}}_{\lambda \lambda \lambda \lambda}, \\
\tilde{\tilde{h}}_{i^{\prime} i}^{\lambda} & =\tilde{\tilde{v}}_{i^{\prime} \lambda i \lambda}, \\
\delta \tilde{\tilde{h}}_{i^{\prime} i} & =\sum_{k k^{\prime}} \tilde{\tilde{v}}_{i^{\prime} k^{\prime} i k} \delta \rho_{k k^{\prime}} .
\end{aligned}
$$

We see that the first-order rearrangement terms (42a) become fully absorbed in the s.p. energies, which are now, as usual, the eigenvalues of mean fields $\tilde{h}^{A}$. Moreover, both the polarizing fields $\tilde{\tilde{h}}^{\lambda}$ and RPA matrices $A$ and $B$ [see Eqs. (23) and (24)] must now be determined using the second-order rearrangement terms (42b). Therefore, owing to the fact that the effective two-body matrix elements (42a) are not antisymmetric, the SI term (44b),

$$
E_{\mathrm{SI}}^{\lambda}=\frac{1}{2} \tilde{\tilde{h}}_{\lambda \lambda}^{\lambda},
$$

is nonzero, and explicitly appears in Eq. (43). This leads to corrections to s.p. energies now having the form,

$$
\delta e_{\lambda}= \pm \delta E= \pm\left(\delta E_{\mathrm{SIF}}^{\lambda}+E_{\mathrm{SI}}^{\lambda}\right)
$$

where, based on the analogy with Eq. (37), the first term can be called self-interaction-free (SIF) polarization correction,

$$
\delta E_{\mathrm{SIF}}^{\lambda}=-\sum_{\omega>0} \frac{\left|\sum_{\mathrm{ph}} \tilde{\tilde{h}}_{\mathrm{ph}}^{\lambda *} X_{\mathrm{ph}}^{\omega}+\tilde{\tilde{h}}_{\mathrm{ph}}^{\lambda} Y_{\mathrm{ph}}^{\omega}\right|^{2}}{\hbar \omega} .
$$

The second-order mean fields $\tilde{\tilde{h}}_{i^{\prime} i}^{\lambda}(44 \mathrm{c})$ and $\delta \tilde{\tilde{h}}_{i^{\prime} i}(44 \mathrm{~d})$ are simply related to the linearized first-order mean fields; that is,

$$
\begin{gathered}
\tilde{\tilde{h}}_{i^{\prime} i}^{\lambda}=\left.\sum_{k^{\prime} k} \frac{\partial \tilde{h}_{i^{\prime} i}}{\partial \rho_{k^{\prime} k}}\right|_{\rho=\rho^{A}} \rho_{k^{\prime} k}^{\lambda}, \\
\delta \tilde{\tilde{h}}_{i^{\prime} i}=\left.\sum_{k^{\prime} k} \frac{\partial \tilde{h}_{i^{\prime} i}}{\partial \rho_{k^{\prime} k}}\right|_{\rho=\rho^{A}} \delta \rho_{k^{\prime} k} .
\end{gathered}
$$

These expressions can be explicitly verified directly from definitions (42). They are extremely useful in practical applications because (i) the second-order mean fields (48a) that define the polarization vertex (47) can be determined without explicitly calculating the second derivatives of matrix elements, (ii) the amplitude mean fields (48b) are the only objects that one has to calculate when using the iterative methods to solve the RPA equations [37], and (iii) exactly the same piece of code can be used to calculate both mean fields (48a) and (48b).

\section{The self-interaction}

The SI term (45), where a particle interacts with the mean field generated by itself, is unphysical, because, in reality, each nucleon should interact with the other nucleons only. As discussed in Sec. II A 2, for an EDF generated by Hamiltonian, no SI appears. However, EDFs generated by density-dependent interactions do produce the SI.

An EDF has a one-body SI if it gives nonzero energy for a single nucleon state. This was discussed in Ref. [35], where it has been shown how the one-body SI of a Skyrme EDF can be removed by introducing extra constraints between the Skyrme coupling constants. We note here that in our approach there is no SI of this type, because we use the so-called "native" time-odd terms [27], that is, those originating from the meanfield averaging of the Skyrme force. In general, the SI results from the violation of the antisymmetry of effective matrix elements (42b); that is, the SI studied here originates from the density dependence of the Skyrme force. Another source of the $\mathrm{SI}$ is the mismatch between the ph and the pp matrix elements of the interaction; see Eq. (68) below. 
For density functionals used in electronic structure calculations, an SI correction was originally introduced by Perdew and Zunger [38], and numerous variations and improvements of the method were later constructed. A short review of the various SI-correction methods used in electronic structure calculations can be found in Ref. [39]. In a nuclear-physics context, the SI problem in connection with density-dependent Skyrme interactions was early on discussed in Ref. [40] and more recently in Ref. [41]. In Refs. [42,43], the SI problem and ways to correct it were discussed in detail, together with the related concept of self pairing; see also Sec. II C.

Within the Skyrme EDF approach without pairing, the SI results from the zero-range density-dependent interaction and from the Coulomb exchange, which is treated in the Slater approximation. Because in the polarization correction (46) the SI term appears explicitly, one can simply remove it from this expression and thus obtain SIF result (47). However, we stress here that the self-consistent calculations performed in odd nuclei do contain the SI term and lead to the polarization correction (46) with the SI term included.

\section{Polarization corrections with pairing}

For an even-paired system corresponding to the average number of particles $A$, one diagonalizes the quasiparticle Hamiltonian $\mathcal{H}^{A}$, which in the standard double-dimension representation [32] reads

$$
\mathcal{H}^{A}=\mathcal{T}+\mathcal{G}^{A}-\lambda \mathcal{N}=\left(\begin{array}{cc}
h^{A}-\lambda & \Delta^{A} \\
-\Delta^{A *} & -h^{A *}+\lambda
\end{array}\right),
$$

where

$$
\begin{aligned}
\mathcal{T} & =\left(\begin{array}{cc}
t & 0 \\
0 & -t^{*}
\end{array}\right), \quad \mathcal{G}^{A}=\left(\begin{array}{cc}
\Gamma^{A} & \Delta^{A} \\
-\Delta^{A *} & -\Gamma^{A *}
\end{array}\right), \\
\mathcal{N} & =\left(\begin{array}{cc}
1 & 0 \\
0 & -1
\end{array}\right),
\end{aligned}
$$

and where $\Gamma_{i^{\prime} i}^{A}$ and $\Delta_{i^{\prime} k^{\prime}}^{A}$ are the ph and pp mean potentials, respectively. The even-system Fermi energy $\lambda$ is adjusted so as the average particle number equals to $\operatorname{Tr} \rho^{A}=A$. In what follows, for clarity we write only one Fermi energy $\lambda$, generalizations to separate neutron and proton Fermi energies being obvious.

The eigenequation for $\mathcal{H}^{A}$ defines one-quasiparticle states $\mathcal{U}_{L}$ and one-quasiparticle energies $E_{L}$,

$$
\mathcal{H}^{A} \mathcal{U}_{L}=E_{L} \mathcal{U}_{L}
$$

where positive (negative) indices $L>0(L<0)$ correspond to positive (negative) quasiparticle energies $E_{L}>0\left(E_{L}<0\right)$ of quasiparticle (quasihole) states. Then the basic dynamical quantity describing the system is the generalized density matrix, $\left(\mathcal{R}^{A}\right)^{2}=\mathcal{R}^{A}$,

$$
\mathcal{R}^{A}=\sum_{L<0} \mathcal{U}_{L} \mathcal{U}_{L}^{+}=\left(\begin{array}{cc}
\rho^{A} & \kappa^{A} \\
-\kappa^{A *} & 1-\rho^{A *}
\end{array}\right),
$$

which projects states on the space of occupied quasihole states,

$$
\mathcal{R}^{A} \mathcal{U}_{L}= \begin{cases}0 & \text { for } \quad L>0 \\ \mathcal{U}_{L} & \text { for } \quad L<0\end{cases}
$$

and depends on the density matrix $\rho^{A}$ and pairing tensor $\kappa^{A}$. When the quasiparticle and quasihole states are arranged as columns of matrix $\mathcal{U}$ in doubled dimensions, they form the matrix of the Bogoliubov transformation [32],

$$
\mathcal{U}=\left(\begin{array}{ll}
U & V^{*} \\
V & U^{*}
\end{array}\right),
$$

in terms of which we have $\rho^{A}=V^{*} V^{T}$ and $\kappa^{A}=V^{*} U^{T}$.

The generalized density matrix of an odd system $\mathcal{R}^{A \pm 1}$ is obtained by the blocking procedure $[32,44]$, whereupon one occupied quasihole state for $L=-\Lambda<0$ is replaced by its empty quasiparticle partner for $L=\Lambda>0$. Then Eqs. (49)-(52) are solved self-consistently again, with the odd-system Fermi energy $\lambda_{A \pm 1}$ adjusted so as the average particle number now equals $\operatorname{Tr} \rho^{A \pm 1}=A \pm 1$. The QRPA polarization correction $\delta \mathcal{R}$ is defined in analogy with Eq. (2) as

$$
\mathcal{R}^{A \pm 1}=\mathcal{R}^{A}+\mathcal{R}^{\Lambda}+\delta \mathcal{R},
$$

where the generalized density matrix $\mathcal{R}^{\Lambda}$ describes the blocked orbital [see Eq. (52)],

$$
\mathcal{R}^{\Lambda}=-\mathcal{U}_{-\Lambda} \mathcal{U}_{-\Lambda}^{+}+\mathcal{U}_{\Lambda} \mathcal{U}_{\Lambda}^{+} .
$$

This gives the density matrix and pairing tensor of the odd system in the form

$$
\begin{gathered}
\rho^{A \pm 1}=\rho^{A}+\rho^{\Lambda}+\delta \rho, \\
\kappa^{A \pm 1}=\kappa^{A}+\kappa^{\Lambda}+\delta \kappa,
\end{gathered}
$$

with explicit contributions coming from the blocked orbital given by

$$
\begin{aligned}
& \rho_{\alpha \beta}^{\Lambda}=-V_{\alpha \Lambda}^{*} V_{\beta \Lambda}+U_{\alpha \Lambda} U_{\beta \Lambda}^{*}, \\
& \kappa_{\alpha \beta}^{\Lambda}=-V_{\alpha \Lambda}^{*} U_{\beta \Lambda}+U_{\alpha \Lambda} V_{\beta \Lambda}^{*} .
\end{aligned}
$$

Density matrices and pairing tensors, self-consistently calculated in even and odd systems, determine the corresponding total energies with pairing as

$$
\begin{aligned}
E^{A} & =\operatorname{Tr} t \rho^{A}+\frac{1}{2} \operatorname{Tr}\left(\Gamma^{A} \rho^{A}-\Delta^{A} \kappa^{A *}\right) \\
& =\frac{1}{2} \operatorname{Tr} \mathcal{T} \mathcal{Q}^{A}+\frac{1}{4} \operatorname{Tr} \mathcal{G}^{A} \mathcal{Q}^{A} \\
E^{A \pm 1} & =\operatorname{Tr} t \rho^{A \pm 1}+\frac{1}{2} \operatorname{Tr}\left(\Gamma^{A \pm 1} \rho^{A \pm 1}-\Delta^{A \pm 1} \kappa^{A \pm 1 *}\right) \\
& =\frac{1}{2} \operatorname{Tr} \mathcal{T} \mathcal{Q}^{A \pm 1}+\frac{1}{4} \operatorname{Tr} \mathcal{G}^{A \pm 1} \mathcal{Q}^{A \pm 1},
\end{aligned}
$$

where $\mathcal{Q}^{A} \equiv \mathcal{R}^{A}-\left(\begin{array}{ll}0 & 0 \\ 0 & 1\end{array}\right)$ and $\mathcal{Q}^{A \pm 1} \equiv \mathcal{R}^{A \pm 1}-\left(\begin{array}{ll}0 & 0 \\ 0 & 1\end{array}\right)$.

Let us now discuss the case of the ph and pp potentials being determined by averaging the corresponding ph and $\mathrm{pp}$ two-body antisymmetric interaction matrix elements, that is,

$$
\begin{aligned}
\Gamma_{i^{\prime} i}^{A} & =\sum_{k^{\prime} k} \bar{v}_{i^{\prime} k^{\prime} i k}^{\mathrm{ph}} \rho_{k k^{\prime}}^{A}, \\
\Delta_{i^{\prime} k^{\prime}}^{A} & =\frac{1}{2} \sum_{i k} \bar{v}_{i^{\prime} k^{\prime} i k}^{\mathrm{pp}} \kappa_{i k}^{A},
\end{aligned}
$$


with the analogous equations defining the potentials in the odd system, $\Gamma^{A \pm 1}$ and $\Delta^{A \pm 1}$. In most nuclear-physics applications, the ph and pp matrix elements are different, which means that the total energies do not, strictly speaking, correspond to an average value of a Hamiltonian.

To discuss the structure of the resulting expressions, we first analyze the situation of these matrix elements being densityindependent, that is, with the rearrangement terms ignored. Then, the potentials depend linearly on densities, and each term in the generalized density matrix $\mathcal{R}^{A \pm 1}$ (55) gives the corresponding term in the generalized potential $\mathcal{G}^{A \pm 1}$,

$$
\mathcal{G}^{A \pm 1}=\mathcal{G}^{A}+\mathcal{G}^{\Lambda}+\delta \mathcal{G} .
$$

By inserting Eqs. (55) and (61) into the expression for the total energy of the odd system (59b), we easily obtain the analog of Eq. (18) with pairing, that is,

$$
\begin{aligned}
E^{A \pm 1}= & E^{A}+\operatorname{Tr}\left[\frac{1}{2}\left(\mathcal{H}^{A}+\lambda \mathcal{N}\right) \mathcal{R}^{\Lambda}+\frac{1}{2}\left(\mathcal{H}^{A}+\lambda \mathcal{N}\right) \delta \mathcal{R}\right. \\
& \left.+\frac{1}{4} \mathcal{H}^{\Lambda} \mathcal{R}^{\Lambda}+\frac{1}{2} \mathcal{H}^{\Lambda} \delta \mathcal{R}+\frac{1}{4} \delta \mathcal{H} \delta \mathcal{R}\right]
\end{aligned}
$$

where, to keep notation consistent with the unpaired case of Sec. II A 2, we have denoted $\mathcal{H}^{\Lambda} \equiv \mathcal{G}^{\Lambda}$ and $\delta \mathcal{H} \equiv \delta \mathcal{G}$.

At this point, taking the trace of Eq. (57a) we can calculate the average number of particles in the blocked state, which gives

$$
\begin{aligned}
A \pm 1 & =A+\operatorname{Tr} \rho^{\Lambda}+\operatorname{Tr} \delta \rho \\
& =A+\frac{1}{2} \operatorname{Tr} \mathcal{N} \mathcal{R}^{\Lambda}+\frac{1}{2} \operatorname{Tr} \mathcal{N} \delta \mathcal{R} .
\end{aligned}
$$

Then we obtain that

$$
\begin{aligned}
E^{A \pm 1}= & E^{A} \pm \lambda+\operatorname{Tr}\left[\frac{1}{2} \mathcal{H}^{A} \mathcal{R}^{\Lambda}+\frac{1}{2} \mathcal{H}^{A} \delta \mathcal{R}\right. \\
& \left.+\frac{1}{4} \mathcal{H}^{\Lambda} \mathcal{R}^{\Lambda}+\frac{1}{2} \mathcal{H}^{\Lambda} \delta \mathcal{R}+\frac{1}{4} \delta \mathcal{H} \delta \mathcal{R}\right]
\end{aligned}
$$

From this point on, derivations proceed exactly as in the case of no pairing (Sec. II A), so we only repeat principal definitions and results. We assume that the blocked quasiparticle wave function $\mathcal{U}_{\Lambda}$, which is determined in the odd system, is identical to that determined in the even system. Only under such an assumption we have $\frac{1}{2} \operatorname{Tr} \mathcal{H}^{A} \mathcal{R}^{\Lambda}=E_{\Lambda}$ [see Eqs. (51) and (56)] and the analog of Eq. (10a) holds,

$$
\delta \mathcal{R}=\mathcal{R}^{A} \delta \mathcal{R}+\delta \mathcal{R} \mathcal{R}^{A}
$$

In Eq. (64) we identify the SI term, analogous to that derived without pairing (45), namely,

$$
E_{\text {SI }}^{\Lambda}=\frac{1}{4} \operatorname{Tr} \mathcal{H}^{\Lambda} \mathcal{R}^{\Lambda}=\frac{1}{2} \operatorname{Tr}\left(\Gamma^{\Lambda} \rho^{\Lambda}-\Delta^{\Lambda} \kappa^{\Lambda *}\right),
$$

where $\Gamma^{\Lambda}$ and $\Delta^{\Lambda}$ are the mean fields generated by the blocked quasiparticle,

$$
\begin{aligned}
\Gamma_{i^{\prime} i}^{\Lambda} & =\sum_{k^{\prime} k} \bar{v}_{i^{\prime} k^{\prime} i k}^{\mathrm{ph}} \rho_{k k^{\prime}}^{\Lambda}, \\
\Delta_{i^{\prime} k^{\prime}}^{\Lambda} & =\frac{1}{2} \sum_{i k} \bar{v}_{i^{\prime} k^{\prime} i k}^{\mathrm{pp}} \kappa_{i k}^{\Lambda} .
\end{aligned}
$$

Thus, the SI term corresponds to the blocked quasiparticle $\Lambda$ that interacts with the generalized mean-field potential it has generated. By combining Eqs. (58) and (67), we can easily derive that

$$
E_{\mathrm{SI}}^{\Lambda}=\sum_{i^{\prime} k^{\prime} i k} U_{k^{\prime} \Lambda}^{*} V_{i^{\prime} \Lambda}\left(\bar{v}_{i^{\prime} k^{\prime} i k}^{\mathrm{pp}}-\bar{v}_{i^{\prime} k^{\prime} i k}^{\mathrm{ph}}\right) U_{k \Lambda} V_{i \Lambda}^{*},
$$

where the antisymmetry of matrix elements $\bar{v}_{i^{\prime} k^{\prime} i k}^{\mathrm{pp}}$ and $\bar{v}_{i^{\prime} k^{\prime} i k}^{\mathrm{ph}}$ was used. We explicitly see that a nonzero value of $E_{\mathrm{SI}}^{\Lambda}$ can only appear when the pp and ph interactions, which define the EDF with pairing, are not identical to one another.

Another assumption we have to make is that the mean field $\mathcal{H}^{\Lambda}$, generated by the blocked quasiparticle $\Lambda$, is appropriately small, of the first order in the small-amplitude expansion. Then, the polarization corrections to paired energies of odd states $\delta E$ and those to quasiparticle energies $\delta E_{\Lambda}$ [cf. Eqs. (20) and (21)], which are defined by

$E^{A \pm 1}=E^{A} \pm \lambda+E_{\Lambda}+\delta E=E^{A} \pm \lambda+\left(E_{\Lambda}+\delta E_{\Lambda}\right)$,

can be expressed in the form

$$
\begin{aligned}
\delta E= & \delta E_{\Lambda}=\frac{1}{2}\left(Z^{*}, \quad Z\right)\left(\begin{array}{cc}
A & B \\
B^{*} & A^{*}
\end{array}\right)\left(\begin{array}{c}
Z \\
Z^{*}
\end{array}\right) \\
& +\left(Z^{*}, \quad Z\right)\left(\begin{array}{c}
W^{\Lambda} \\
W^{\Lambda *}
\end{array}\right)+E_{\mathrm{SI}}^{\Lambda},
\end{aligned}
$$

where $Z$ and $W^{\Lambda}$ represent vectors of quasiparticle-quasihole matrix elements of $\delta \mathcal{R}$ and $\mathcal{H}^{\Lambda}$, respectively; that is,

$$
\begin{aligned}
Z_{L L^{\prime}} & =\mathcal{U}_{L}^{+} \delta \mathcal{R} \mathcal{U}_{-L^{\prime}}, \\
W_{L L^{\prime}}^{\Lambda} & =\mathcal{U}_{L}^{+} \mathcal{H}^{\Lambda} \mathcal{U}_{-L^{\prime}},
\end{aligned}
$$

for $L>L^{\prime}>0$, and $A$ and $B$ are the standard components of the QRPA matrix [32].

An equation for $Z$ can easily be derived by following the steps presented in Sec. II A 3; it simply results from the requirement that the correction to the energy (70) is stationary, which gives

$$
\left(\begin{array}{cc}
A & B \\
B^{*} & A^{*}
\end{array}\right)\left(\begin{array}{c}
Z \\
Z^{*}
\end{array}\right)=-\left(\begin{array}{c}
W^{\Lambda} \\
W^{\Lambda *}
\end{array}\right)
$$

and

$$
\delta E=\delta E_{\mathrm{SIF}}^{\Lambda}+E_{\mathrm{SI}}^{\Lambda}
$$

for

$$
\delta E_{\mathrm{SIF}}^{\Lambda}=-\frac{1}{2}\left(W^{\Lambda *}, \quad W^{\Lambda}\right)\left(\begin{array}{cc}
A & B \\
B^{*} & A^{*}
\end{array}\right)^{-1}\left(\begin{array}{c}
W^{\Lambda} \\
W^{\Lambda *}
\end{array}\right) .
$$

The QRPA SIF polarization correction to quasiparticle energy explicitly reads

$$
\delta E_{\mathrm{SIF}}^{\Lambda}=-\sum_{\omega>0} \frac{\left|\sum_{L>L^{\prime}}\left(W_{L L^{\prime}}^{\Lambda *} X_{L L^{\prime}}^{\omega}+W_{L L^{\prime}}^{\Lambda} Y_{L L^{\prime}}^{\omega}\right)\right|^{2}}{\hbar \omega}
$$

where $X_{L L^{\prime}}^{\omega}$ and $Y_{L L^{\prime}}^{\omega}$ are the standard QRPA amplitudes.

\section{A few remarks to Sec. II}

Before discussing numerical results in the next section, let us briefly touch upon the problem of conserved symmetries. 
No specific conserved symmetry was, in fact, assumed in the derivations presented so far. In the Appendix, we discuss in detail implications of conserving the spherical symmetry. For the conserved parity, the s.p. wave function $\psi_{\lambda}(\boldsymbol{r})$ of a polarizing state $\lambda$ has a definite parity $\pi_{\lambda}$, that is, $\psi_{\lambda}(-\boldsymbol{r})=$ $\pi_{\lambda} \psi_{\lambda}(\boldsymbol{r})$. Therefore, the density matrix $\rho^{\lambda}$, which in the space coordinates reads $\rho^{\lambda}\left(\boldsymbol{r}, \boldsymbol{r}^{\prime}\right)=\psi_{\lambda}(\boldsymbol{r}) \psi_{\lambda}\left(\boldsymbol{r}^{\prime}\right)^{*}$, is always parity even, irrespective of the parity of the polarizing state: $\rho^{\lambda}\left(-\boldsymbol{r},-\boldsymbol{r}^{\prime}\right)=\pi_{\lambda}^{2} \psi_{\lambda}(\boldsymbol{r}) \psi_{\lambda}\left(\boldsymbol{r}^{\prime}\right)^{*}=\rho^{\lambda}\left(\boldsymbol{r}, \boldsymbol{r}^{\prime}\right)$. With pairing correlations included, analogous arguments hold for quasiparticle wave function $\mathcal{U}_{-\Lambda}$ and generalized density matrix $\mathcal{R}^{\Lambda}$. Because interactions are parity invariant, the positive parity of $\rho^{\lambda}$ or $\mathcal{R}^{\Lambda}$ implies positive parity of mean fields $h^{\lambda}$ or $W^{\Lambda}$, and thus only positive-parity phonons contribute to the energy shifts in Eqs. (47) or (76).

This means that, for conserved spherical and parity symmetries, all polarization corrections discussed in Sec. II relate to the RPA and QRPA channels and phonons $J^{\pi}$ with positive parity $\pi=+1$ only. Therefore, the discussed polarization corrections cannot, and do not, involve any couplings to negative-parity channels, including those to the very important $3^{-}$channel. The latter can only be treated within the PVC methods [14], which will be discussed in a forthcoming study [45].

We also note that the polarization corrections studied in this work are equivalent to the "diagonal" approximation to the PVC; that is, they involve the PVC energy denominators $\left(\epsilon_{p}-\epsilon_{p^{\prime}}-\hbar \omega\right)^{-1}$ for $p=p^{\prime}$; see Eqs. (47) and (76). The "diagonal" approximation has two important consequences. First, the polarization vertex is limited to the same state as the one for which the PVC is evaluated. This shows again that the parity conservation in the vertex excludes coupling to negative-parity phonons. Second, polarization corrections do not include large PVC contributions from states with $\left(\epsilon_{p} \simeq \epsilon_{p^{\prime}}+\hbar \omega\right)$, which correspond to small energy denominators. Thus, the lack of couplings to negative-parity channels constitutes the main drawback of the mean-field methods in describing states in odd nuclei, and the "diagonal" approximation may imply missing large corrections to their binding energies.

There is a hypothetical possibility of including the coupling to negative-parity phonons, namely, through a dynamical parity breaking of the mean field. This would require performing generator-coordinate-like calculations based on mixing octupole-deformed states for odd nuclei. It is, however, unclear if such an approach can be equivalent to the PVC that includes negative-parity phonons.

Another drawback of the mean-field approach, clearly identified in Secs. II B and II C, is the presence of the SI terms in the mean-field binding energies of odd nuclei. Based on the analyses performed within the RPA and QRPA methods, we could explicitly identify these terms, which allows for calculating them after variation. The explicit identification may also allow us to remove them before variation, which will be the subject of subsequent studies. Of course, although not explicitly "visible," the SI terms are also present in the mean-field binding energies of even nuclei, and in the future new functionals with these terms removed should also be studied.
We note that expression (68) for the SI energy with pairing is valid only for density-independent interactions. However, expression (66) does take effects of density-dependent interactions into account, provided it is evaluated for mean-field potentials with rearrangement terms included, as derived in Sec. II A; that is,

$$
E_{\mathrm{SI}}^{\Lambda}=\frac{1}{2} \operatorname{Tr}\left(\tilde{\tilde{h}}^{\Lambda} \rho^{\Lambda}-\Delta^{\Lambda} \kappa^{\Lambda *}\right)
$$

Let us now summarize our results on the equivalence between the $\mathrm{HF}(\mathrm{B})$ and $(\mathrm{Q}) \mathrm{RPA}$ polarization corrections. As we showed in Sec. II B for the HF case, this equivalence has the form of equation [cf. Eq. (43)]:

$$
E^{A \pm 1}-E^{A} \mp e_{\lambda}-E_{\mathrm{SI}}^{\lambda}=\delta E_{\mathrm{SIF}}^{\lambda} .
$$

On the left-hand side, there appear quantities calculated within the HF method, that is, (i) the total self-consistent ground-state energies of odd and even systems (38), $E^{A \pm 1}$ and $E^{A}$, respectively; (ii) HF s.p. energy $e_{\lambda}$ (44a); and (iii) SI energy $E_{\mathrm{SI}}^{\lambda}$ (45). On the right-hand side, there appears the SIF correction $\delta E_{\mathrm{SIF}}^{\lambda}$ (47), calculated within the RPA method.

Similarly, in Sec. II C we showed for the HFB case that [cf. Eq. (69)]

$$
E^{A \pm 1}-E^{A} \mp \lambda-E_{\Lambda}-E_{\mathrm{SI}}^{\Lambda}=\delta E_{\mathrm{SIF}}^{\Lambda},
$$

where on the left-hand side there appear quantities calculated within the HFB method, that is, (i) the total self-consistent paired ground-state energies of odd and even systems (60), $E^{A \pm 1}$ and $E^{A}$, respectively; (ii) HFB even-system Fermi energy $\lambda$; (iii) HFB one-quasiparticle energy $E_{\Lambda}$ (51); and (iv) SI energy $E_{\mathrm{SI}}^{\Lambda}$ (77). On the right-hand side, there appears the SIF correction $\delta E_{\mathrm{SIF}}^{\Lambda}(76)$, calculated within the QRPA method.

The above equivalence between the $\mathrm{HF}(\mathrm{B})$ and $(\mathrm{Q}) \mathrm{RPA}$ polarization corrections holds only up to the validity of the small-amplitude expansion (9), which was used before in the standard derivations presented in Refs. [28-31]. However, in this work we have identified additional assumptions required for Eqs. (78) and (79) to hold, namely,

(i) differences between the s.p. (one-quasiparticle) states calculated in even and odd systems must be neglected;

(ii) the density matrix $\rho^{\lambda}\left(\mathcal{R}^{\Lambda}\right)$, corresponding to the polarizing s.p. (one-quasiparticle) state, must be appropriately small, that is, of the first order in the small-amplitude expansion (9);

(iii) the mean field $h^{\lambda}\left(\mathcal{H}^{\Lambda}\right)$, corresponding to the polarizing s.p. (one-quasiparticle) state, must be appropriately small, that is, of the first order in the small-amplitude expansion;

(iv) differences between the density-dependent matrix elements in odd and even systems can be accounted for by the small-amplitude expansion up to second order.

These assumptions can only be tested by numerical calculations and in Sec. III are shown to be quite well justified.

An important observation relates to the size of the phase space taken into account after solving the (Q)RPA equations. In the full PVC calculations, one usually includes only those phonons that are both significantly collective and have suitably 
low energies [14]. However, assuming the above assumptions are fulfilled, the equivalence of the $\mathrm{HF}(\mathrm{B})$ and (Q)RPA polarization corrections is obtained only when all phonons are included, irrespective of their collectivity or energy. This is because, when deriving the SIF corrections of Eqs. (47) or (76), the exact (Q)RPA completeness relations must be used. In addition, the same equivalence guarantees the convergence of sums in Eqs. (47) or (76); indeed, with the increasing phase space, owing to the variational principle, the $\mathrm{HF}(\mathrm{B})$ energies must converge, and thus the (Q)RPA polarization corrections must converge too.

\section{RESULTS}

All calculations presented in this section aim at comparing self-consistent results obtained using the deformed solver HFODD (v2.52k) [46], with RPA and QRPA solutions implemented in the spherical solver HOSPHE [47]. We used the configuration space that includes all harmonic-oscillator shells up to $N_{0}=15$.

\section{A. RPA calculations in ${ }^{100} \mathrm{Sn}$ for the Skyrme EDF SV}

We begin the presentation by showing examples of calculations performed for the case of an exact HF approximation, as discussed in Sec. II A. To this end, we employed the density-independent Skyrme interaction SV [48] and we analyzed results only for neutrons, so as to avoid effects of density-dependent Slater approximation for the Coulomb exchange term. On the one hand, to treat the EDF SV as fully generated by an interaction, we included in the functional all tensor terms, that is, those given by the square of the spin-orbit density $\boldsymbol{J}^{2}$, which were originally neglected [48]. Also the so-called "native" time-odd terms [27], that is, those originating from the mean-field averaging of the Skyrme force, were all included. On the other hand, as mentioned in Sec. II A, we neglected the so-called center-of-mass correction to the kinetic energy.

In Fig. 1 we test Eq. (21); that is, we compare polarization corrections,

$$
\delta e_{\lambda}= \pm\left(E^{A \pm 1}-E^{A}\right)-e_{\lambda}
$$

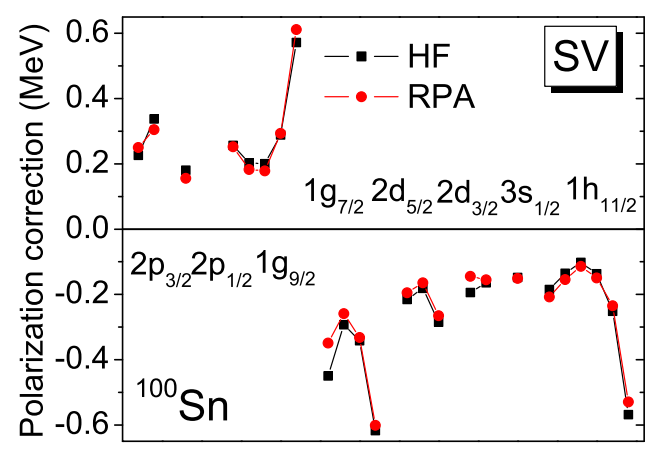

FIG. 1. (Color online) Comparison of polarization corrections of selected orbitals in ${ }^{100} \mathrm{Sn}$, determined using the HF and RPA methods and Skyrme EDF SV [48]; see text. Lines connect the values obtained for different projections of the angular momentum $\left|m_{\lambda}\right|=\frac{1}{2}, \ldots, j_{\lambda}$ (from left to right).
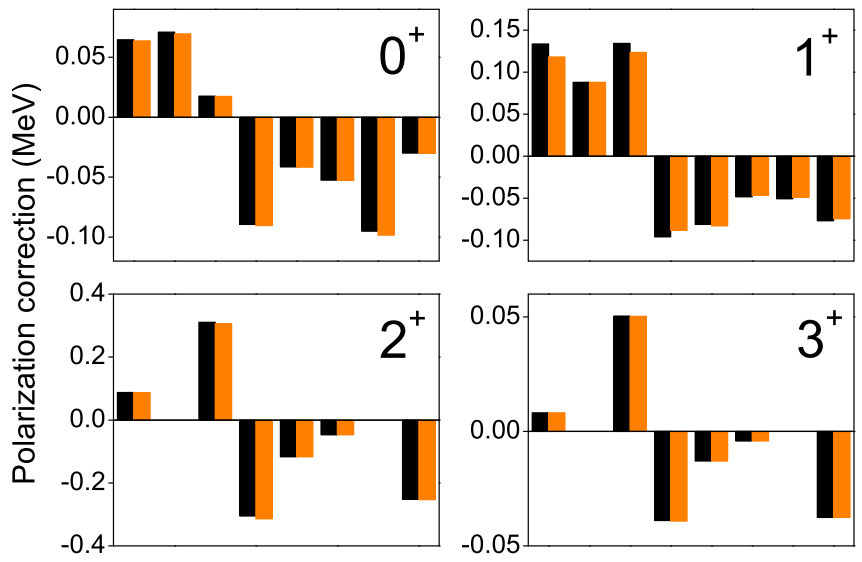

FIG. 2. (Color online) Polarization corrections of $\left|m_{\lambda}\right|=j_{\lambda}$ orbitals in ${ }^{100} \mathrm{Sn}$, determined by not including (left bars) and including (right bars) the orbital-dependent terms in the RPA matrices; see text. The order of orbitals is the same as shown in Fig. 1. Contributions coming from four RPA channels $J^{\pi}=0^{+}, 1^{+}, 2^{+}$, and $3^{+}$are shown separately (note very different scales).

obtained from the HF energies of odd and even systems, $E^{A \pm 1}$ and $E^{A}$, and HF s.p. energies, $e_{\lambda}$, with those determined form the RPA solutions [Eq. (37)]. Apart from a few cases, the obtained agreement is nearly perfect. This result is particularly gratifying, because it confirms not only the analytical derivations presented in Sec. II A and the Appendix, but also the validity of two completely independent numerical codes.

At this point, we must discuss one important aspect of the $\mathrm{HF}$ calculations in odd nuclei. In principle, for any given value of $m_{\lambda}$, there may exist two solutions: one with prolate and another one with oblate shape. Usually only the lowest one can be converged; the other one, being excited, either does not converge or falls down to the lowest one. In our calculations, in full agreement with the standard Nilsson diagram [32], we obtain converged prolate (oblate) solutions for low- $m_{\lambda}$ (high$m_{\lambda}$ ) particle states, and vice versa for the hole states. We note here that we did not constrain these solutions to axial symmetry; nevertheless, stable triaxial solutions were never obtained.

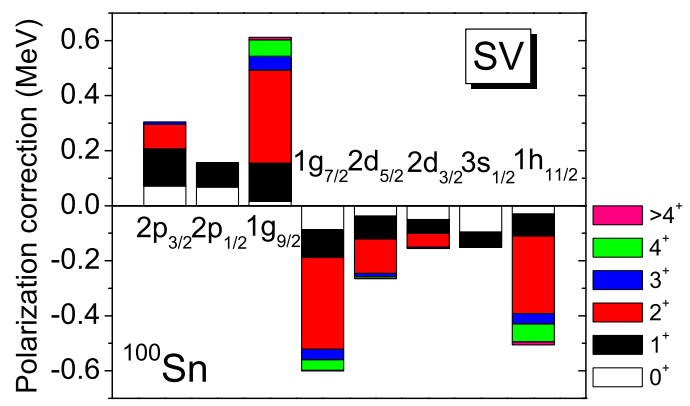

FIG. 3. (Color online) Contributions to polarization corrections of $\left|m_{\lambda}\right|=j_{\lambda}$ orbitals in ${ }^{100} \mathrm{Sn}$, coming from different $J^{\pi}$ RPA channels, determined for the Skyrme EDF SV [48]. The order of orbitals is the same as shown in Fig. 1. Contributions are ordered according to the value of $J$, with the $0^{+}$channels shown nearest the abscissa. 


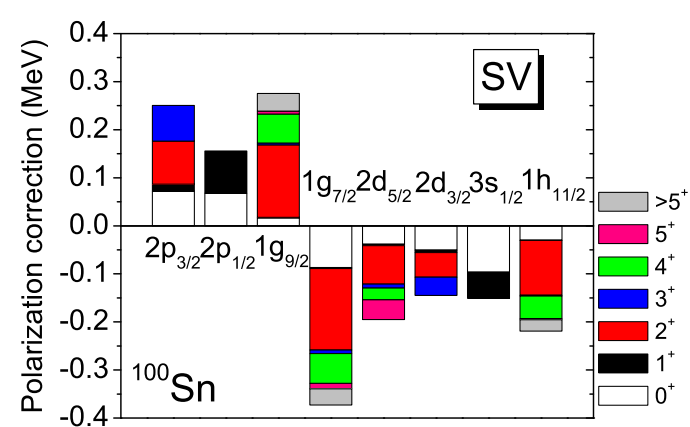

FIG. 4. (Color online) Same as in Fig. 3, but for the $\left|m_{\lambda}\right|=\frac{1}{2}$ orbitals.

Next we tested the assumption, discussed in Sec. II A, related to the smallness of terms $\rho^{\lambda}$ and $h^{\lambda}$ with respect to the small-amplitude expansion. In Fig. 2, we compare polarization corrections determined using the standard RPA matrices (24b) with those containing the orbital-dependent terms (24a). Because both sets of results are almost identical, we conclude that in medium-heavy nuclei like ${ }^{100} \mathrm{Sn}$, the orbital-dependent terms can be safely ignored. This significantly simplifies the calculations because a single common solution of the RPA equation can then be used to determine polarization corrections for all orbitals.

In Fig. 3, we show polarization corrections of the $\left|m_{\lambda}\right|=j_{\lambda}$ orbitals in ${ }^{100} \mathrm{Sn}$, split into contributions from different $J^{\pi}$ RPA channels. First we note that the geometric constraints in Eq. (A16) limit the polarizations of $j_{\lambda}$ orbitals to channels with $J \leqslant 2 j_{\lambda}$. As expected, the largest contributions come from the coupling to the quadrupole channel $2^{+}$; however, the monopole $0^{+}$and dipole $1^{+}$channels also significantly contribute. For higher- $j_{\lambda}$ orbitals, channels $3^{+}$and $4^{+}$show some effect, whereas channels with $J>4$ can be safely neglected. For the $\left|m_{\lambda}\right|=\frac{1}{2}$ orbitals shown in Fig. 4 , the convergence is slightly slower, but still all terms with $J>5$ contribute very little.

\section{B. RPA calculations in ${ }^{100} \mathrm{Sn}$ for the Skyrme EDF SLy5}

We now proceed to discuss the problem of SI energies in the EDF calculations, presented in Sec. IIB. To this

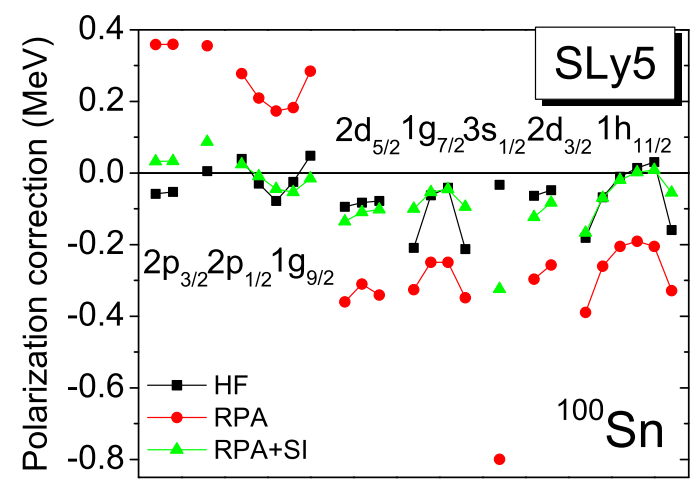

FIG. 5. (Color online) Same as in Fig. 1, but for the Skyrme EDF SLy5 [49]. The RPA results correspond to the SIF terms in Eq. (46), whereas RPA + SI denotes both SIF and SI contributions combined.

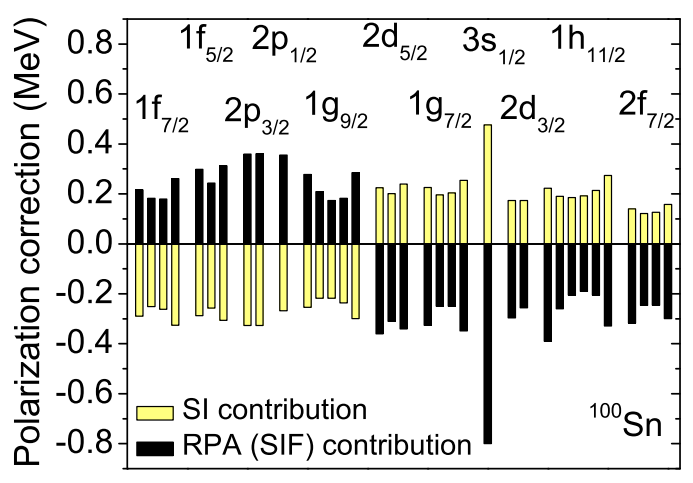

FIG. 6. (Color online) The SIF and SI contributions to the polarization corrections of Eq. (46), calculated in ${ }^{100} \mathrm{Sn}$ for the Skyrme EDF SLy5.

end, we repeated the self-consistent calculations presented in Sec. III A by employing the Skyrme EDF SLy5 [49]. This is a standard Skyrme parametrization containing a strong densitydependent term, for which we can study the SI energies, as defined in Eq. (45). As before, the "native" time-odd terms of SLy5 were included and the center-of-mass correction to the kinetic energy was neglected.

In Fig. 5, we show the RPA (SIF) contributions to polarization corrections (46), and we compare the total polarization corrections calculated using Eq. (46) with the HF results (80). The obtained agreement is very good, although not as perfect as that obtained in Sec. III A for the Skyrme EDF SV. Moreover, the RPA results obtained for the SV and SLy5 functionals are significantly different from one another, the latter ones being close to about $\pm 0.4 \mathrm{MeV}$ for holes and particles, respectively. We also see that the SLy5 results are much less $m_{\lambda}$ dependent.

The most striking observation seen in Fig. 5, also explicitly illustrated in Fig. 6, is a strong cancellation between the SIF and SI contributions to the polarization corrections (46). This cancellation makes the HF polarization corrections quite small and gives the explanation to the long-standing problem of significant differences between the magnitudes of the HF and RPA values [20]. Indeed, it is the unphysical SI contribution that renders the HF polarization corrections so small; see Ref. [25] for a set of comprehensive calculations across the mass chart.

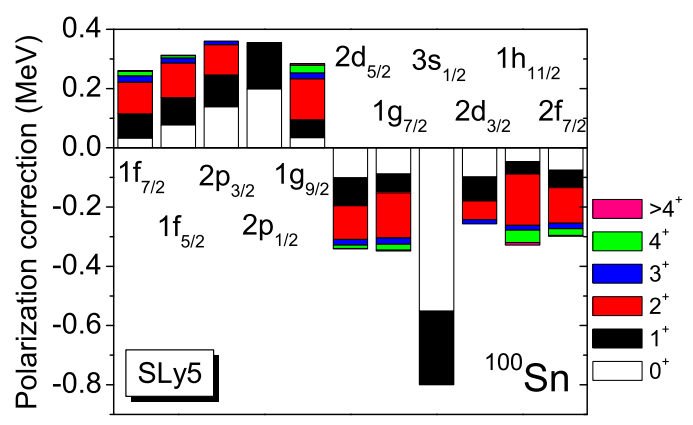

FIG. 7. (Color online) Same as in Fig. 3, but for the contributions to the RPA SIF polarization corrections of $\left|m_{\lambda}\right|=j_{\lambda}$ orbitals, determined for the Skyrme EDF SLy5. 


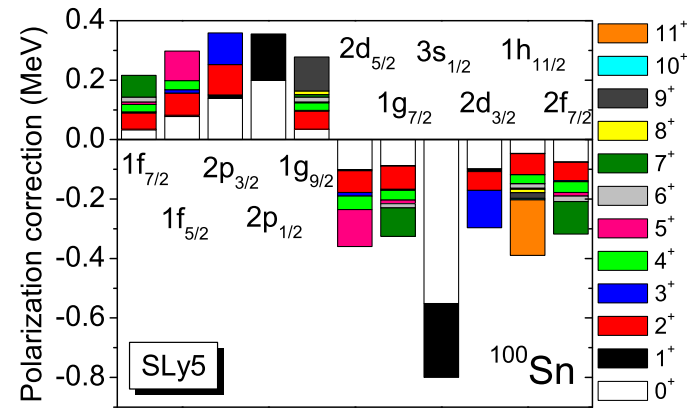

FIG. 8. (Color online) Same as in Fig. 7, but for the $\left|m_{\lambda}\right|=\frac{1}{2}$ orbitals.

We conclude this section by showing, in Figs. 7 and 8 , the RPA SIF polarization corrections of the $\left|m_{\lambda}\right|=j_{\lambda}$ and $\left|m_{\lambda}\right|=\frac{1}{2}$ orbitals, respectively, split into contributions coming from different $J^{\pi}$ RPA channels and calculated in ${ }^{100} \mathrm{Sn}$ for the Skyrme EDF SLy5. These can be compared with the analogous ones shown in Figs. 3 and 4 for the Skyrme EDF SV. We first see that for the $\left|m_{\lambda}\right|=j_{\lambda}$ orbitals, the convergence patterns obtained for both EDFs are fairly similar. However, for the $\left|m_{\lambda}\right|=\frac{1}{2}$ orbitals, contributions coming from the $J=2 j_{\lambda}$ phonons turn out to be always quite large. For example, results obtained for the $h_{11 / 2}$ orbital certainly require taking into account the $J=11^{+}$phonons.

\section{QRPA calculations in ${ }^{100-132}$ Sn for the Skyrme EDF SLy5}

To present numerical results pertaining to the description of polarization effects with pairing correlations included (Sec. II C), we performed HFB and QRPA calculations for the tin isotopes ${ }^{100-132} \mathrm{Sn}$. As in Sec. III B, we used the Skyrme EDF SLy5, whereas the pairing interaction was modeled by a contact volume pairing force with the strength of $V_{0}=$ $200 \mathrm{MeV} \mathrm{fm}^{-3}$ and active pairing space restricted to states below $60 \mathrm{MeV}$.

In Fig. 9 we aim at testing Eq. (69), where $E^{A \pm 1}$ and $E^{A}$ are self-consistent HFB ground-state energies of odd and even nuclei, respectively, $\lambda$ and $E_{\Lambda}$ are the HFB Fermi energy and quasiparticle energy of the blocked state, respectively, and $\delta E$ is the QRPA (SIF $+\mathrm{SI}$ ) polarization correction (74). For this comparison, we must decide whether to use the HFB results obtained for the lighter $(A-1)$ or heavier $(A+1)$ odd system. Obviously, the former (latter) option must be used

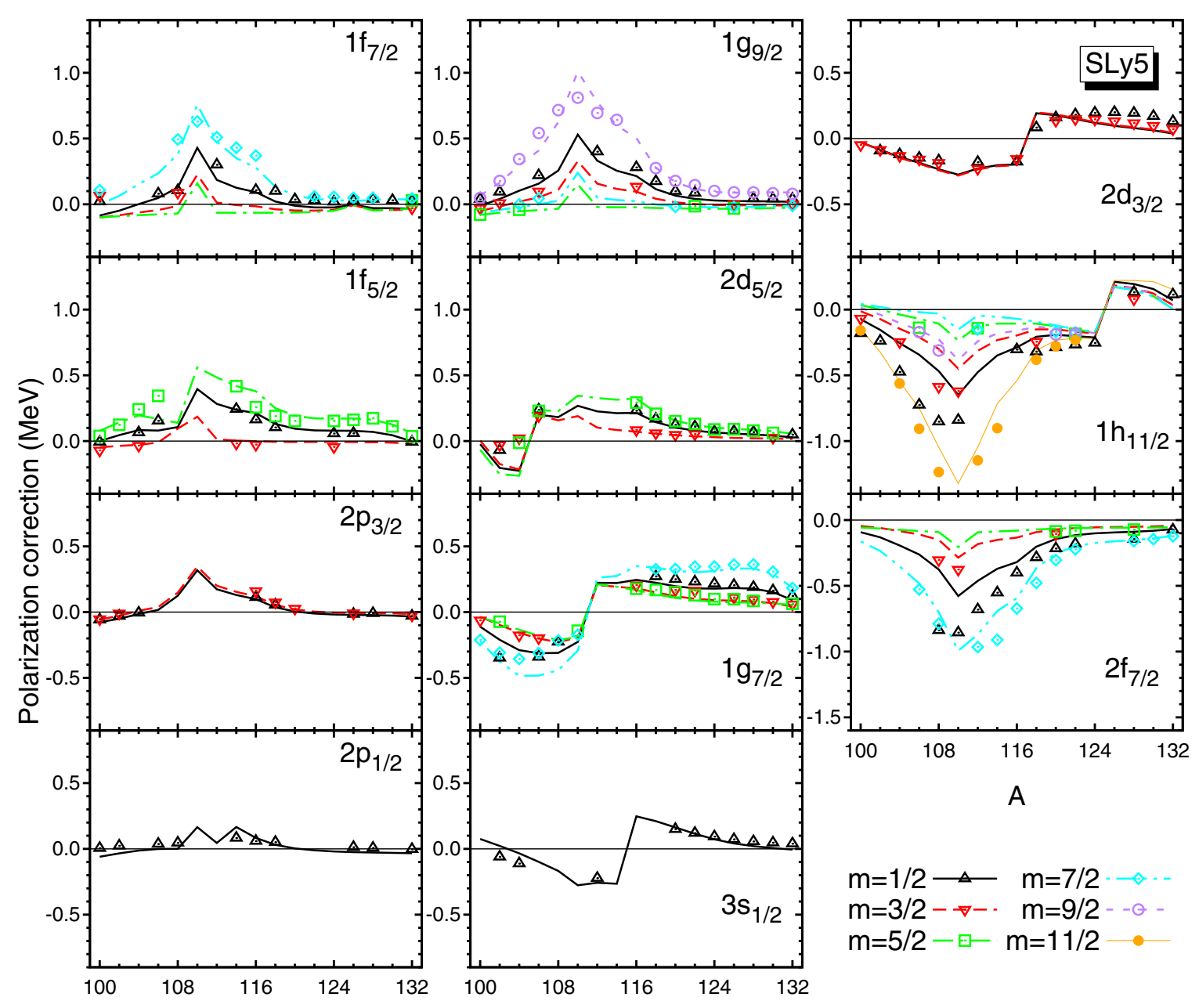

FIG. 9. (Color online) Comparison of the QRPA SIF + SI (symbols) and HFB (lines) polarization corrections to quasiparticle energies in tin isotopes. 


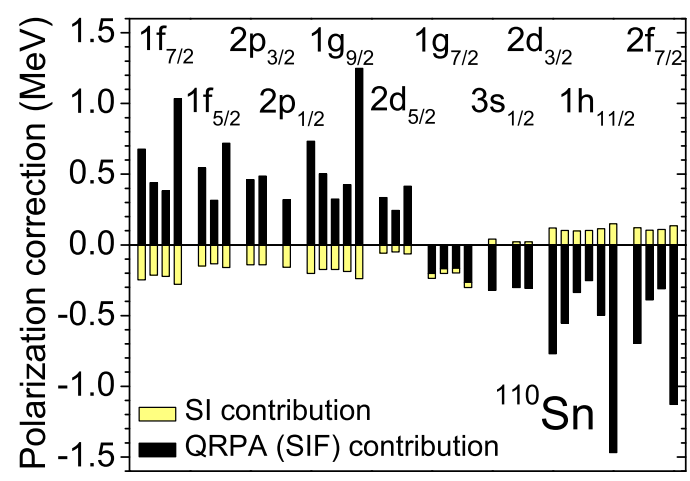

FIG. 10. (Color online) Same as in Fig. 6, but for ${ }^{110} \mathrm{Sn}$.

for predominantly hole-type (particle-type) quasiparticles. For quasiparticles near the Fermi level, however, there is a certain degree of ambiguity, which we here arbitrarily resolve by checking whether the s.p. energy $e_{\Lambda}$ corresponding to the blocked quasiparticle state is below or above the Fermi level $\lambda$. In practice, we determine $e_{\Lambda}$ by diagonalizing in the even nucleus the mean-field Hamiltonian $h^{A}$, which is a part of the HFB Hamiltonian (49). In addition, to link results presented in this section to those presented before for magic nuclei without pairing, in Figs. 9-16 we plot results for hole states with flipped signs, that is,

$$
\begin{aligned}
& -\delta E=+\left(E^{A}-E^{A-1}\right)-\left(\lambda-E_{\Lambda}\right) \text { for } e_{\Lambda}<\lambda \text {, } \\
& +\delta E=-\left(E^{A}-E^{A+1}\right)-\left(\lambda+E_{\Lambda}\right) \text { for } e_{\Lambda}>\lambda
\end{aligned}
$$

[cf. Eq. (80)].

Within such a convention, in Fig. 9 we show the QRPA SIF + SI (symbols) and HFB (lines) polarization corrections given by the left-hand and right-hand sides of Eqs. (81), respectively. We note that not all blocked quasiparticle states could be converged in all studied nuclei, and thus in the figure there is quite a number of missing HFB points. Nevertheless, we conclude that the agreement between the QRPA and the HFB results is satisfactory. By this we establish the equivalence of the two methods in determining the polarization corrections with pairing.

In Figs. 10 and 11, we compare the QRPA SIF (76) and SI (77) contributions to the polarization corrections. Similar

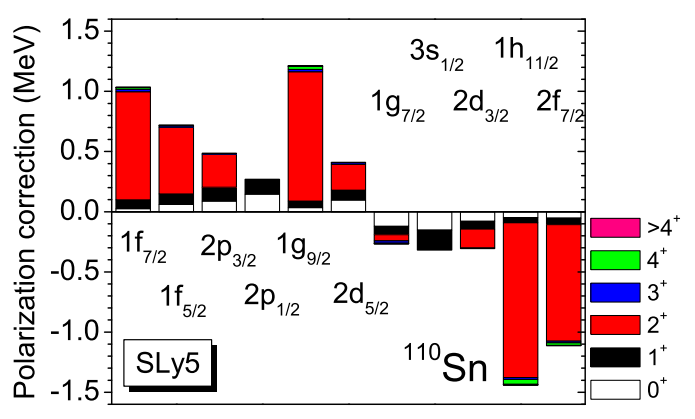

FIG. 12. (Color online) Same as in Fig. 7, but for ${ }^{110} \mathrm{Sn}$.

to that seen in the case without pairing, shown in Fig. 6, the SIF and SI terms always have opposite signs, and thus the SI partially cancels the SIF contribution. However, here the SI terms are relatively smaller, and thus they to a lesser degree decrease the SIF contributions, as compared to the results with no pairing. It is fairly difficult to pin down specific reasons for the qualitative differences between the SI energies obtained with and without pairing correlations. It could be that the SI energies related to density dependence of the Skyrme interaction (45) and those related to differences between the pp and ph channels (68), partially cancel out.

Convergence of the QRPA polarization corrections as a function of the angular momentum $J$ of the QRPA phonons, shown in Figs. 12 and 13, is much faster than that without pairing; cf. Figs. 7 and 8 . Here, the $2^{+}$channels clearly dominate. This can be interpreted as the result of the quadrupole collectivity being increased by the pairing correlations. In most cases, channels with $J>4$ can be safely neglected, with the exception of the $J=2 j_{\Lambda}$ channels that slightly contribute to the corrections of the $m_{\Lambda}=\frac{1}{2}$ quasiparticle states.

All results presented up to now pertain to single-reference $\mathrm{HF}(\mathrm{B})$ and (Q)RPA calculations; that is, only one single orbital, with a fixed projection $m_{\lambda}$ or $m_{\Lambda}$, was occupied and was inducing polarization effects. As discussed previously, this required symmetry breaking in the $\mathrm{HF}(\mathrm{B})$ solutions and required coupling of (Q)RPA phonons to odd particles in a symmetry-nonconserving way. However, a symmetryconserving (Q)RPA coupling [14] simply amounts to averaging the results obtained for different values of $m_{\lambda}$ or $m_{\Lambda}$; see Eqs. (A18). In Figs. 14-16, we present results for the averages obtained in this way.

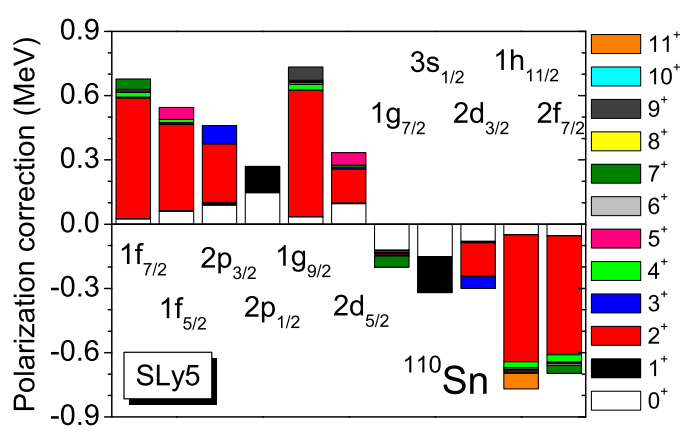

FIG. 13. (Color online) Same as in Fig. 8, but for ${ }^{110} \mathrm{Sn}$.

FIG. 11. (Color online) Same as in Fig. 6, but for ${ }^{120} \mathrm{Sn}$. 


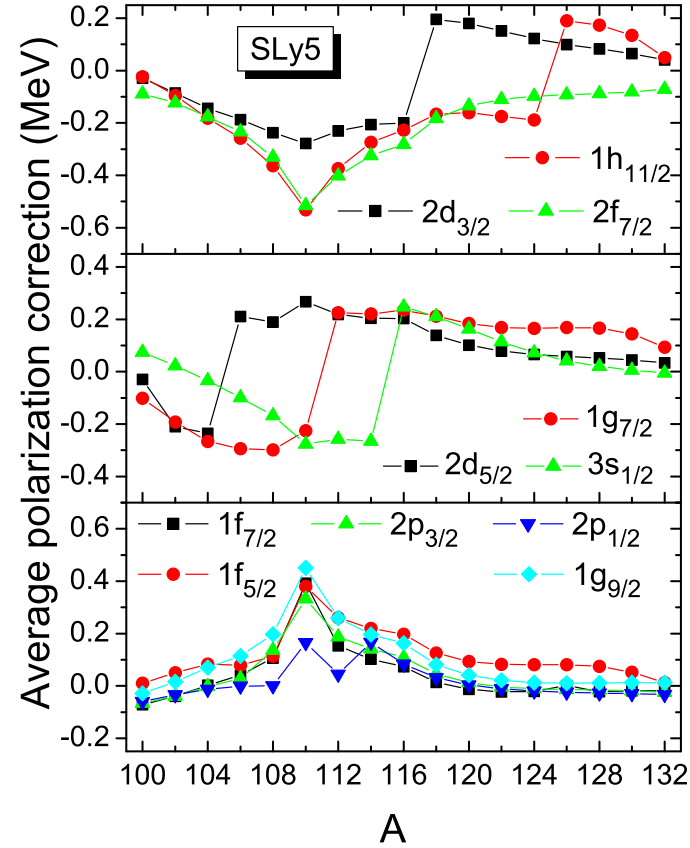

FIG. 14. (Color online) Average QRPA $(\mathrm{SIF}+\mathrm{SI})$ polarization corrections $\delta E_{\mathrm{SIF}}+E_{\mathrm{SI}}$, Eqs. (A18), in tin isotopes.

Figures 14 and 15 summarize our results obtained for the QRPA polarization effects in tin isotopes. We see that the polarization corrections strongly depend on $A$. This is mostly attributable to the fact that for the Skyrme EDF SLy5, the quadrupole collectivity in tin isotopes varies with $A$ and peaks near $A=110$, where the nuclei are softest against the quadrupole deformation and the QRPA $2^{+}$phonons are lowest

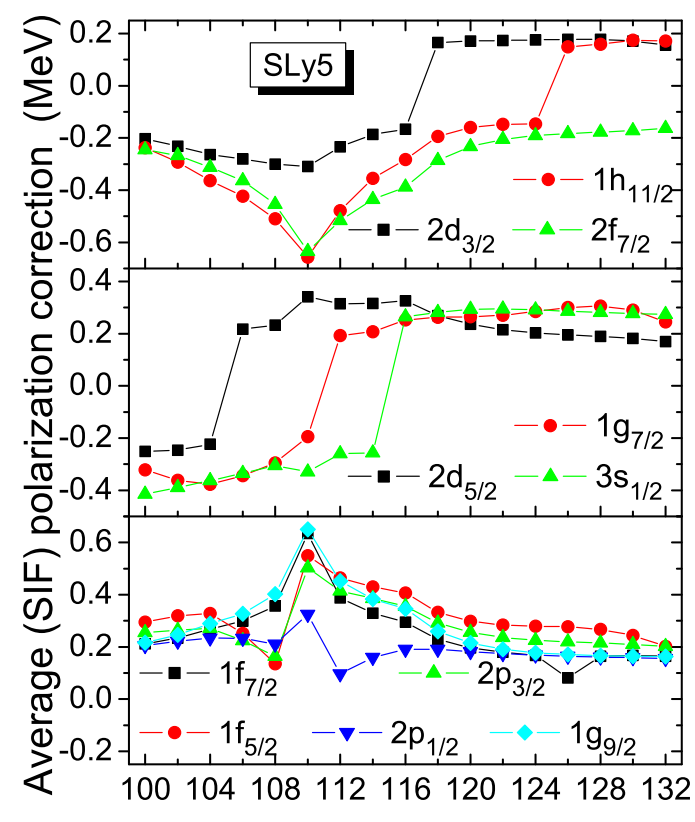

A

FIG. 15. (Color online) Same as in Fig. 14, but for the QRPA SIF polarization corrections only.

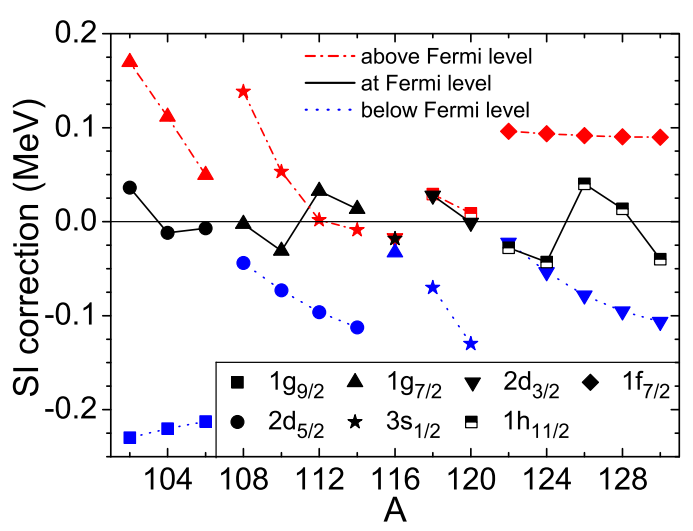

FIG. 16. (Color online) Average SI corrections $E_{\mathrm{SI}}$ (A18a) plotted for quasiparticles closest to the Fermi level in open-shell tin isotopes.

in energy and have the largest strength. At $A=110$, values of polarization corrections reach up to $0.6 \mathrm{MeV}$.

Note that when a given orbital crosses the Fermi level, its plotted polarization correction jumps from negative to positive values, which is the result of the plotting convention explained in Eqs. (81). In fact, the QRPA polarization corrections to quasiparticle energies are always negative. For the SIF contributions [cf. Eqs. (76) and (A16)], this fact is obvious, whereas for paired open-shell nuclei, smaller opposite-sign SI contributions are unable to change this general rule.

In Fig. 16, for selected quasiparticles in open-shell tin isotopes, we show values of the average SI corrections $E_{\mathrm{SI}}$ (A18a). Solid lines connect values obtained for quasiparticles at the Fermi level, and dashed and dotted lines pertain to those just below and above the Fermi level, respectively. It is interesting to see that for the quasiparticle at the Fermi level, the SI corrections become fairly small, not exceeding $50 \mathrm{keV}$, whereas away from the Fermi level they can be of the order of $200 \mathrm{keV}$; see also Figs. 10 and 11. If this observation is confirmed or derived in a systematic way, we can hope that near ground states of odd open-shell nuclei, the effects of SI energies might be small. This is important, because the odd-even mass staggering, where masses of odd nuclei enter, is routinely used to gauge the intensity of pairing correlations.

\section{CONCLUSIONS}

In the present study, we investigated links between the mean-field and polarization-correction approaches to masses of odd nuclei. The former ones are rooted in the EDF methods and strive to describe odd systems by blocking odd particle or quasiparticle states. Energies of odd nuclei are then obtained by minimization methods, in full analogy with those used in even nuclei, and by employing the same EDFs. The latter ones are based on the perturbative "diagonal" coupling between the odd particle and vibrational phonons calculated in even systems.

Following the classic analyses presented in Refs. [28-31], we derived links between these two classes of approaches also in the case of density-dependent interactions and/or paired systems. This allowed us to show limitations of the polarization-correction methods as compared to the full 
"nondiagonal" PVC that is rooted in the many-body perturbation theory.

We performed numerical analyses using the deformed mean-field code HFODD (v2.52k) [46], which is able to solve self-consistent equations in odd nuclei by breaking all symmetries. The polarization corrections were independently calculated using the spherical code HOSPHE [47], which has the capability to solve efficiently the (Q)RPA equations. The comparison of results allowed us to identify the reason of discrepancies between the masses of odd nuclei calculated with these two approaches, which turns out to be the self-interaction energy, polluting the mean-field energies of odd nuclei. Our derivations also allowed us to explicitly define and calculate the self-interaction energies, which then can be subtracted from the mean-field results leading to SIF masses.

\section{ACKNOWLEDGMENTS}

Fruitful and inspiring discussions with Gianluca Colò are gratefully acknowledged. This work was supported in part by the THEXO JRA within the EU-FP7-IA Project ENSAR (Grant No. 262010), Academy of Finland and University of Jyväskylä within the FIDIPRO program, Polish National Science Center under Contract No. 2012/07/B/ST2/03907, and Bulgarian Science Fund under Contract No. NuPNETSARFEN DNS7RP01/0003. We acknowledge the CSC-IT Center for Science Ltd., Finland, for the allocation of computational resources.

\section{APPENDIX: SPHERICAL SYMMETRY}

In this Appendix, we specify the final equations obtained for the SI energy (66) and polarization correction (76) to the case of spherical symmetry, for which the numerical analyses of this work were performed. First we note that the use of spherical symmetry does not mean that in the odd system the spherical symmetry is conserved.

Indeed, in the even system, the quasiparticles move in the spherical field and thus their wave functions are characterized by quantum numbers $\Lambda \equiv n_{\Lambda} j_{\Lambda} m_{\Lambda}$ that comprise the principal quantum number $n_{\Lambda}$, angular momentum $j_{\Lambda}$, and its projection $m_{\Lambda}$. Quasiparticles having different projections $m_{\Lambda}$ are degenerate, and, therefore, any linear combination of them can be used as the blocked orbital. In this work, we make a simplifying assumption that the blocked quasiparticle corresponds to a specific single value of the projection $m_{\Lambda}$. The general case could have been treated equally easy, and at the end of the Appendix we discuss the meaning of it.

An odd state, obtained by blocking a quasiparticle, becomes necessarily deformed. In the calculations performed with the deformed code HFODD, this is particularly well and explicitly visible, as the self-consistent solutions obtained in odd systems always acquire small but nonzero deformations. The aim of this appendix is to show in which way the deformation, and the dependence of final results on the values of $m_{\Lambda}$, appears in the QRPA calculations that are performed in the spherical basis and using the spherical code HOSPHE.
We begin by specifying expressions for the density matrix and pairing tensor of the blocked quasiparticle (58) to the case of the spherical basis $\alpha \equiv n_{\alpha} j_{\alpha} m_{\alpha}$,

$$
\begin{aligned}
\rho_{n_{\alpha} j_{\alpha} m_{\alpha}, n_{\beta} j_{\beta} m_{\beta}}^{\Lambda}= & -V_{n_{\alpha} j_{\alpha} m_{\alpha}, n_{\Lambda} j_{\Lambda} m_{\Lambda}}^{*} V_{n_{\beta} j_{\beta} m_{\beta}, n_{\Lambda} j_{\Lambda} m_{\Lambda}} \\
& +U_{n_{\alpha} j_{\alpha} m_{\alpha}, n_{\Lambda} j_{\Lambda} m_{\Lambda}} U_{n_{\beta} j_{\beta} m_{\beta}, n_{\Lambda} j_{\Lambda} m_{\Lambda}}^{*}, \\
\kappa_{n_{\alpha} j_{\alpha} m_{\alpha}, n_{\beta} j_{\beta} m_{\beta}}^{\Lambda}= & -V_{n_{\alpha} j_{\alpha} m_{\alpha}, n_{\Lambda} j_{\Lambda} m_{\Lambda}}^{*} U_{n_{\beta} j_{\beta} m_{\beta}, n_{\Lambda} j_{\Lambda} m_{\Lambda}} \\
& +U_{n_{\alpha} j_{\alpha} m_{\alpha}, n_{\Lambda} j_{\Lambda} m_{\Lambda}} V_{n_{\beta} j_{\beta} m_{\beta}, n_{\Lambda} j_{\Lambda} m_{\Lambda}}^{*},
\end{aligned}
$$

where the spherically symmetric quasiparticle wave functions read

$$
\begin{aligned}
& U_{n_{\alpha} j_{\alpha} m_{\alpha}, n_{L} j_{L} m_{L}}=\delta_{j_{\alpha} j_{L}} \delta_{m_{\alpha} m_{L}} U_{n_{\alpha} n_{L}}^{j_{L}}, \\
& V_{n_{\alpha} j_{\alpha} m_{\alpha}, n_{L} j_{L} m_{L}}=(-1)^{j_{\alpha}-m_{\alpha}} \delta_{j_{\alpha} j_{L}} \delta_{m_{\alpha},-m_{L}} V_{n_{\alpha} n_{L}}^{j_{L}},
\end{aligned}
$$

and $U_{n_{\alpha} n_{L}}^{j_{L}}$ and $V_{n_{\alpha} n_{L}}^{j_{L}}$ are solutions of the HFB equation, obtained for the quasiparticle state with quantum numbers $L \equiv n_{L} j_{L} m_{L}$ in the given $j_{L}$ block.

In a way similar to that for the angular-momentumprojected deformed states [32], we can write the deformed density matrix $\rho^{\Lambda}$ and pairing tensor $\kappa^{\Lambda}$ as sums of those projected on good angular momentum $J$ and its projections on the laboratory axis $M$ and on the intrinsic axis $K, \rho^{\Lambda, J M K}$ and $\kappa^{\Lambda, J M K}$, that is,

$$
\begin{aligned}
& \rho_{n_{\alpha} j_{\alpha} m_{\alpha}, n_{\beta} j_{\beta} m_{\beta}}^{\Lambda}=\sum_{J K} \rho_{n_{\alpha} j_{\alpha} m_{\alpha}, n_{\beta} j_{\beta} m_{\beta}}^{\Lambda, J K K}, \\
& \kappa_{n_{\alpha} j_{\alpha} m_{\alpha}, n_{\beta} j_{\beta} m_{\beta}}^{\Lambda}=\sum_{J K} \kappa_{n_{\alpha} j_{\alpha} m_{\alpha}, n_{\beta} j_{\beta} m_{\beta}}^{\Lambda, J K K},
\end{aligned}
$$

where only the $M=K$ terms appear in the expansion [32]. Using the Wigner-Eckart theorem [50], one can always express laboratory spherical-tensor matrices, $\rho^{\Lambda, J M K}$ and $\kappa^{\Lambda, J M K}$, corresponding to quantum numbers $J M$, as

$$
\begin{aligned}
\rho_{n_{\alpha} j_{\alpha} m_{\alpha}, n_{\beta} j_{\beta} m_{\beta}}^{\Lambda, J M K}= & \frac{1}{\sqrt{2 j_{\alpha}+1}} C_{j_{\beta} m_{\beta} J M}^{j_{\alpha} m_{\alpha}}\left\langle n_{\alpha} j_{\alpha}\left\|\rho^{\Lambda, J K}\right\| n_{\beta} j_{\beta}\right\rangle, \\
\kappa_{n_{\alpha} j_{\alpha} m_{\alpha}, n_{\beta} j_{\beta} m_{\beta}}^{\Lambda, J M K}= & \frac{1}{\sqrt{2 j_{\alpha}+1}}(-1)^{j_{\beta}-m_{\beta}} C_{j_{\beta},-m_{\beta} J M}^{j_{\alpha} m_{\alpha}} \\
& \times\left\langle n_{\alpha} j_{\alpha} \| \kappa^{\Lambda, J K}|| n_{\beta} j_{\beta}\right\rangle,
\end{aligned}
$$

where the reduced matrix elements can be calculated as

$$
\begin{aligned}
\left\langle n_{\alpha} j_{\alpha}\left\|\rho^{\Lambda, J K}\right\| n_{\beta} j_{\beta}\right\rangle= & \sum_{m_{\alpha} m_{\beta}} \frac{2 J+1}{\sqrt{2 j_{\alpha}+1}} C_{j_{\beta} m_{\beta} J K}^{j_{\alpha} m_{\alpha}} \\
& \times \rho_{n_{\alpha} j_{\alpha} m_{\alpha}, n_{\beta} j_{\beta} m_{\beta}}^{\Lambda}, \\
\left\langle n_{\alpha} j_{\alpha}\left\|\kappa^{\Lambda, J K}\right\| n_{\beta} j_{\beta}\right\rangle= & \sum_{m_{\alpha} m_{\beta}} \frac{2 J+1}{\sqrt{2 j_{\alpha}+1}} C_{j_{\beta} m_{\beta} J K}^{j_{\alpha} m_{\alpha}} \\
& \times(-1)^{j_{\beta}+m_{\beta}} \kappa_{n_{\alpha} j_{\alpha} m_{\alpha}, n_{\beta} j_{\beta},-m_{\beta}}^{\Lambda} .
\end{aligned}
$$


Validity of expansions (A3) can now be explicitly verified using summation properties of the Clebsh-Gordan coefficients [50].

At this point, we can use the fact that the spherical-basis properties of mean fields are exactly the same as those of densities; that is, Eqs. (A3)-(A5) hold equally well for $\rho^{\Lambda}$ and $\kappa^{\Lambda}$ replaced by $\Gamma^{\Lambda}$ and $\Delta^{\Lambda}$, respectively. Then, by summing the Clebsh-Gordan coefficients again, traces in Eq. (66) can be explicitly evaluated, which gives

$$
\begin{aligned}
E_{\mathrm{SI}}^{\Lambda}= & \frac{1}{2} \sum_{n_{\alpha} j_{\alpha} n_{\beta} j_{\beta} J K} \frac{1}{2 J+1} \\
& \times\left(\left\langle n_{\alpha} j_{\alpha} \| \Gamma^{\Lambda, J K}|| n_{\beta} j_{\beta}\right\rangle\left\langle n_{\alpha} j_{\alpha} \| \rho^{\Lambda, J,-K}|| n_{\beta} j_{\beta}\right\rangle^{*}\right. \\
& \left.+\left\langle n_{\alpha} j_{\alpha} \| \Delta^{\Lambda, J K}|| n_{\beta} j_{\beta}\right\rangle\left\langle n_{\alpha} j_{\alpha} \| \kappa^{\Lambda, J K}|| n_{\beta} j_{\beta}\right\rangle^{*}\right) .
\end{aligned}
$$

Similarly, we can evaluate the QRPA SIF polarization correction of Eq. (76). Because the spherical QRPA amplitudes $X$ and $Y$ can be labeled with the good quantum numbers $J M$, we have

$\delta E_{\mathrm{SIF}}^{\Lambda}=-\frac{1}{4} \sum_{J M} \sum_{\omega_{J}>0} \frac{\left|\sum_{L L^{\prime}}\left(W_{L L^{\prime}}^{\Lambda *} X_{L L^{\prime}}^{\omega, J M}+W_{L L^{\prime}}^{\Lambda} Y_{L L^{\prime}}^{\omega, J M}\right)\right|^{2}}{\hbar \omega_{J}}$.

From the Wigner-Eckart theorem, amplitudes $X^{\omega, J M}$ and $Y^{\omega, J M}$ read

$$
\begin{aligned}
X_{n_{L} j_{L} m_{L}, n_{L^{\prime}} j_{L^{\prime}} m_{L^{\prime}}}^{\omega, J}= & \frac{1}{\sqrt{2 j_{L}+1}}(-1)^{j_{L^{\prime}}-m_{L^{\prime}} C_{j_{L^{\prime}},-m_{L^{\prime}} J M}^{j_{L} m_{L}}} \\
& \times\left\langle n_{L} j_{L}\left\|X^{\omega, J}\right\| n_{L^{\prime}} j_{L^{\prime}}\right\rangle, \\
Y_{n_{L} j_{L} m_{L}, n_{L^{\prime}} j_{L^{\prime}} m_{L^{\prime}}}^{\omega, J}= & \frac{1}{\sqrt{2 j_{L}+1}}(-1)^{j_{L}-m_{L}} C_{j_{L^{\prime}} m_{L^{\prime}} J M}^{j_{L}, m_{L}} \\
& \times\left\langle n_{L} j_{L}\left\|Y^{\omega, J}\right\| n_{L^{\prime}} j_{L^{\prime}}\right\rangle .
\end{aligned}
$$

Using the fact that the spherical-basis properties of quasiparticle matrix $W^{\Lambda}$ are the same as those of $\kappa^{\Lambda}$ [see Eqs. (A3b), (A4b), and (A5b)], we can derive that

$$
\begin{aligned}
\delta E_{\mathrm{SIF}}^{\Lambda}= & -\frac{1}{4} \sum_{J K} \frac{1}{(2 J+1)^{2}} \sum_{\omega_{J}>0} \frac{1}{\hbar \omega_{J}} \mid \sum_{n_{L} j_{L} n_{L^{\prime}} j_{L^{\prime}}}\left(\left\langle n_{L} j_{L} \| W^{\Lambda, J K}|| n_{L^{\prime}} j_{L^{\prime}}\right\rangle^{*}\left\langle n_{L} j_{L}\left\|X^{\omega, J}\right\| n_{L^{\prime}} j_{L^{\prime}}\right\rangle\right. \\
& \left.-(-1)^{J+K}\left\langle n_{L} j_{L} \| W^{\Lambda, J,-K}|| n_{L^{\prime}} j_{L^{\prime}}\right\rangle\left\langle n_{L} j_{L} \| Y^{\omega, J}|| n_{L^{\prime}} j_{L^{\prime}}\right\rangle\right)\left.\right|^{2} .
\end{aligned}
$$

Finally, we note that Eqs. (A3)-(A10) hold for an arbitrary blocked quasiparticle. However, when the reduced matrix elements (A5) are evaluated for a specific quasiparticle (A2) that has a fixed value of projection $m_{\Lambda}$, we obtain

$$
\begin{aligned}
& \left\langle n_{\alpha} j_{\alpha} \| \rho^{J K}|| n_{\beta} j_{\beta}\right\rangle=\frac{2 J+1}{\sqrt{2 j_{\Lambda}+1}} C_{j_{\Lambda} m_{\Lambda} J 0}^{j_{\Lambda} m_{\Lambda}} \delta_{j_{\alpha}, j_{\Lambda}} \delta_{j_{\beta}, j_{\Lambda}} \delta_{K 0}\left[-(-1)^{J} V_{n_{\alpha} n_{\Lambda}}^{j_{\Lambda} *} V_{n_{\beta} n_{\Lambda}}^{j_{\Lambda}}+U_{n_{\alpha} n_{\Lambda}}^{j_{\Lambda}} U_{n_{\beta} n_{\Lambda}}^{j_{\Lambda} *}\right], \\
& \left\langle n_{\alpha} j_{\alpha} \| \kappa^{J K}|| n_{\beta} j_{\beta}\right\rangle=\frac{2 J+1}{\sqrt{2 j_{\Lambda}+1}} C_{j_{\Lambda} m_{\Lambda} J 0}^{j_{\Lambda} m_{\Lambda}} \delta_{j_{\alpha}, j_{\Lambda}} \delta_{j_{\beta}, j_{\Lambda}} \delta_{K 0}\left[(-1)^{J} V_{n_{\alpha} n_{\Lambda}}^{j_{\Lambda} *} U_{n_{\beta} n_{\Lambda}}^{j_{\Lambda}}+U_{n_{\alpha} n_{\Lambda}}^{j_{\Lambda}} V_{n_{\beta} n_{\Lambda}}^{j_{\Lambda} *}\right] .
\end{aligned}
$$

In this case, in Eqs. (A6) and (A10) only the $K=0$ terms contribute to the SI energy and SIF polarization correction, respectively.

In any channel $J$, the results depend on $m_{\Lambda}$ only through the Clebsh-Gordan coefficient $C_{j_{\Lambda} m_{\Lambda} J 0}^{j_{\Lambda} m_{\Lambda}}$. It is, therefore, advantageous to define triple reduced matrix elements that do not depend on $m_{\Lambda}$,

$$
\begin{aligned}
& \left\langle\alpha j_{\alpha} \| \rho^{\Lambda, J K}|| \beta j_{\beta}\right\rangle=F\left(m_{\Lambda}, J\right) \delta_{K 0}\left\langle\alpha j _ { \alpha } \left\|\left|\rho^{\Lambda, J} \|\right|\left|\beta j_{\beta}\right\rangle,\right.\right. \\
& \left\langle\alpha j_{\alpha} \| \kappa^{\Lambda, J K}|| \beta j_{\beta}\right\rangle=F\left(m_{\Lambda}, J\right) \delta_{K 0}\left\langle\alpha j_{\alpha} \|\left|\kappa^{\Lambda, J}\right||| \beta j_{\beta}\right\rangle,
\end{aligned}
$$

for

$$
F\left(m_{\Lambda}, J\right)=\sqrt{2 J+1} C_{j_{\Lambda} m_{\Lambda} J 0}^{j_{\Lambda} m_{\Lambda}} .
$$

Then, using the triple reduced matrix elements, calculation of mean fields can be performed only once, and the results valid for arbitrary values of $m_{\Lambda}$ can be reconstructed as

$$
\begin{aligned}
\left\langle\alpha j_{\alpha}\left\|\Gamma^{\Lambda, J K}\right\| \beta j_{\beta}\right\rangle & =F\left(m_{\Lambda}, J\right) \delta_{K 0}\left\langle\alpha j_{\alpha}\left\|\left|\Gamma^{\Lambda, J}\right|\right\| \beta j_{\beta}\right\rangle, \\
\left\langle\alpha j_{\alpha}\left\|\Delta^{\Lambda, J K}\right\| \beta j_{\beta}\right\rangle & =F\left(m_{\Lambda}, J\right) \delta_{K 0}\left\langle\alpha j_{\alpha} \|\left|\Delta^{\Lambda, J}\right||| \beta j_{\beta}\right\rangle, \\
\left\langle\alpha j_{\alpha} \| W^{\Lambda, J K}|| \beta j_{\beta}\right\rangle & =F\left(m_{\Lambda}, J\right) \delta_{K 0}\left\langle\alpha j_{\alpha}\left\|\left|W^{\Lambda, J}\right|\right\| \beta j_{\beta}\right\rangle .
\end{aligned}
$$


In terms of the triple reduced matrix elements, for fixed- $m_{\Lambda}$ quasiparticles, results (A6) and (A10) can be expressed through contributions coming from different channels,

$$
\begin{aligned}
E_{\mathrm{SI}, J}^{\Lambda}= & \frac{1}{2} \sum_{n_{\alpha} n_{\beta}}\left(\left\langle n_{\alpha} j_{\Lambda}||\left|\Gamma^{\Lambda, J}\right||| n_{\beta} j_{\Lambda}\right\rangle\left\langle n_{\alpha} j_{\Lambda}||\left|\rho^{\Lambda, J}\right||| n_{\beta} j_{\Lambda}\right\rangle^{*}+\left\langle n_{\alpha} j_{\Lambda}||\left|\Delta^{\Lambda, J}\right| \mid n_{\beta} j_{\Lambda}\right\rangle\left\langle n_{\alpha} j_{\Lambda} \|\left|\kappa^{\Lambda, J}\right||| n_{\beta} j_{\Lambda}\right\rangle^{*}\right), \\
\delta E_{\mathrm{SIF}, J}^{\Lambda}= & -\frac{1}{4(2 J+1)} \sum_{\omega_{J}>0} \frac{1}{\hbar \omega_{J}} \mid \sum_{n_{L} j_{L} n_{L^{\prime}} j_{L^{\prime}}}\left(\left\langle n_{L} j_{L}||\left|W^{\Lambda, J}\right||| n_{L^{\prime}} j_{L^{\prime}}\right\rangle^{*}\left\langle n_{L} j_{L}\left\|X^{\omega, J}\right\| n_{L^{\prime}} j_{L^{\prime}}\right\rangle\right. \\
& \left.-(-1)^{J}\left\langle n_{L} j_{L}||\left|W^{\Lambda, J}\right||| n_{L^{\prime}} j_{L^{\prime}}\right\rangle\left\langle n_{L} j_{L} \| Y^{\omega, J}|| n_{L^{\prime}} j_{L^{\prime}}\right\rangle\right)\left.\right|^{2},
\end{aligned}
$$

whereupon they read

$$
\begin{gathered}
E_{\mathrm{SI}}^{\Lambda}=\sum_{J}\left(C_{j_{\Lambda} m_{\Lambda} J 0}^{j_{\Lambda} m_{\Lambda}}\right)^{2} E_{\mathrm{SI}, J}^{\Lambda}, \\
\delta E_{\mathrm{SIF}}^{\Lambda}=\sum_{J}\left(C_{j_{\Lambda} m_{\Lambda} J 0}^{j_{\Lambda} m_{\Lambda}}\right)^{2} \delta E_{\mathrm{SIF}, J}^{\Lambda} .
\end{gathered}
$$

We note that factors $\delta_{j_{\alpha}, j_{\Lambda}} \delta_{j_{\beta}, j_{\Lambda}}$, which are present in Eqs. (A11), allowed for reducing Eq. (A15) to terms with $j_{\alpha}=j_{\beta}=j_{\Lambda}$ only. However, fields (A14) are not restricted to $j_{L}=j_{L^{\prime}}=j_{\Lambda}$ and thus the QRPA SIF corrections (A16) must be summed up over $j_{L}$ and $j_{L^{\prime}}$.

From expressions (A17) we see that the polarization corrections calculated for orbitals with given values of $m_{\Lambda}$ are obtained by folding the $J$-dependent (but $m_{\Lambda}$-independent) contributions $E_{\mathrm{SI}, J}^{\Lambda}$ and $\delta E_{\mathrm{SIF}, J}^{\Lambda}$ with simple Clebsh-Gordan coefficients. Values of these coefficients thus dictate how strongly a given channel $J$ contributes. Moreover, owing to the summation properties of the Clebsh-Gordan coefficients, the average contributions read

$$
\begin{gathered}
E_{\mathrm{SI}} \equiv \frac{1}{2 j_{\Lambda}+1} \sum_{m_{\Lambda}} E_{\mathrm{SI}}^{\Lambda}=\sum_{J} \frac{1}{2 J+1} E_{\mathrm{SI}, J}^{\Lambda}, \\
\delta E_{\mathrm{SIF}} \equiv \frac{1}{2 j_{\Lambda}+1} \sum_{m_{\Lambda}} \delta E_{\mathrm{SIF}}^{\Lambda}=\sum_{J} \frac{1}{2 J+1} \delta E_{\mathrm{SIF}, J}^{\Lambda} .
\end{gathered}
$$

As shown in this Appendix, by blocking quasiparticles that have fixed values of projections $m_{\Lambda}$, one obtains only the $K=0$ terms in densities and fields; that is, deformations of odd systems are axial. It is also clear that by blocking quasiparticles with mixed values of $m_{\Lambda}$, one would have obtained nonzero reduced matrix elements for nonzero values of $K$, and thus in odd systems, nonaxial deformations would have appeared. Numerical results presented in this study indicate, however, that axial solutions have systematically lower energies.

[1] W. H. Dickhoff and D. Van Neck, Many-Body Theory Exposed! (World Scientific, Singapore, 2005).

[2] A. Bohr and B.R. Mottelson, Nuclear Structure (W.A. Benjamin, New York, 1975), Vol. II.

[3] I. Hamamoto, Phys. Rep. 10, 63 (1974).

[4] V. Bernard and N. Van Giai, Nucl. Phys. A 348, 75 (1980).

[5] C. Mahaux, P. F. Bortignon, R. A. Broglia, and C. H. Dasso, Phys. Rep. 120, 1 (1985).

[6] S. Galès, C. Stoyanov, and A. Vdovin, Phys. Rep. 166, 125 (1988).

[7] V. Van der Sluys, D. Van Neck, M. Waroquier, and J. Ryckebusch, Nucl. Phys. A 551, 210 (1993).

[8] A. V. Avdeenkov and S. P. Kamerdzhiev, Phys. Lett. B 459, 423 (1999).

[9] S. Baroni, F. Barranco, P. F. Bortignon, R. A. Broglia, G. Colò, and E. Vigezzi, Phys. Rev. C 74, 024305 (2006).
[10] E. Litvinova, P. Ring, and V. Tselyaev, Phys. Rev. C 75, 064308 (2007).

[11] S. Mishev and V. V. Voronov, Phys. Rev. C 78, 024310 (2008).

[12] K. Yoshida, Phys. Rev. C 79, 054303 (2009).

[13] G. Colò and P. Bortignon, Nucl. Phys. A 696, 427 (2001).

[14] G. Colò, H. Sagawa, and P. F. Bortignon, Phys. Rev. C 82, 064307 (2010).

[15] E. V. Litvinova and A. V. Afanasjev, Phys. Rev. C 84, 014305 (2011).

[16] E. Litvinova, Phys. Rev. C 85, 021303 (2012).

[17] K. Mizuyama, G. Colo, and E. Vigezzi, Phys. Rev. C 86, 034318 (2012).

[18] A. Idini, F. Barranco, and E. Vigezzi, Phys. Rev. C 85, 014331 (2012).

[19] V. Somà, C. Barbieri, and T. Duguet, Phys. Rev. C 87, 011303 (2013). 
[20] P. F. Bortignon, G. Colò, and H. Sagawa, J. Phys. G 37, 064013 (2010).

[21] K. Rutz, M. Bender, J. A. Maruhn, P.-G. Reinhard, and W. Greiner, Nucl. Phys. A 634, 67 (1998).

[22] W. Satuła, J. Dobaczewski, and W. Nazarewicz, Phys. Rev. Lett. 81, 3599 (1998).

[23] T. Duguet, P. Bonche, P.-H. Heenen, and J. Meyer, Phys. Rev. C 65, 014310 (2001).

[24] T. Duguet, P. Bonche, P.-H. Heenen, and J. Meyer, Phys. Rev. C 65, 014311 (2001).

[25] M. Zalewski, J. Dobaczewski, W. Satuła, and T. R. Werner, Phys. Rev. C 77, 024316 (2008).

[26] G. F. Bertsch, C. A. Bertulani, W. Nazarewicz, N. Schunck, and M. V. Stoitsov, Phys. Rev. C 79, 034306 (2009).

[27] N. Schunck, J. Dobaczewski, J. McDonnell, J. Moré, W. Nazarewicz, J. Sarich, and M. V. Stoitsov, Phys. Rev. C 81, 024316 (2010).

[28] G. E. Brown, in Facets of Physics, edited by D. A. Bromley and V. W. Hughes (Academic Press, New York, 1970).

[29] J. P. Blaizot and G. Ripka, Quantum Theory of Finite Systems (MIT Press, Cambridge, MA, 1986).

[30] E. Lipparini and S. Stringari, Ann. Phys. 173, 411 (1987).

[31] E. Lipparini and S. Stringari, Phys. Rep. 175, 103 (1989).

[32] P. Ring and P. Schuck, The Nuclear Many-Body Problem (Springer-Verlag, Berlin, 1980).

[33] M. Bender, P.-H. Heenen, and P.-G. Reinhard, Rev. Mod. Phys. 75, 121 (2003).

[34] J. Dobaczewski, B.G. Carlsson, and M. Kortelainen, J. Phys. G: Nucl. Part. Phys. 37, 075106 (2010).

[35] N. Chamel, Phys. Rev. C 82, 061307 (2010).
[36] P.-G. Reinhard and H. Flocard, Nucl. Phys. A 584, 467 (1995).

[37] J. Toivanen, B. G. Carlsson, J. Dobaczewski, K. Mizuyama, R. R. Rodríguez-Guzmán, P. Toivanen, and P. Veselý, Phys. Rev. C 81, 034312 (2010).

[38] J. P. Perdew and A. Zunger, Phys. Rev. B 23, 5048 (1981).

[39] C. Legrand, E. Suraud, and P.-G. Reinhard, J. Phys. B: At. Mol. Opt. Phys. 35, 1115 (2002).

[40] S. Stringari and D. M. Brink, Nucl. Phys. A 304, 307 (1978).

[41] P. Klüpfel, P.-G. Reinhard, T. J. Burvenich, and J. A. Maruhn, Phys. Rev. C 79, 034310 (2009).

[42] D. Lacroix, T. Duguet, and M. Bender, Phys. Rev. C 79, 044318 (2009).

[43] M. Bender, T. Duguet, and D. Lacroix, Phys. Rev. C 79, 044319 (2009).

[44] G. Bertsch, J. Dobaczewski, W. Nazarewicz, and J. Pei, Phys. Rev. A 79, 043602 (2009).

[45] D. Tarpanov et al. (unpublished).

[46] N. Schunck, J. Dobaczewski, J. McDonnell, W. Satuła, J. A. Sheikh, A. Staszczak, M. Stoitsov, and P. Toivanen, Comput. Phys. Commun. 183, 166 (2012).

[47] B. G. Carlsson, J. Toivanen, J. Dobaczewski, P. Veselý, Y. Gao, and D. Ward (to be published).

[48] M. Beiner, H. Flocard, N. Van Giai, and P. Quentin, Nucl. Phys. A 238, 29 (1975).

[49] E. Chabanat, P. Bonche, P. Haensel, J. Meyer, and R. Schaeffer, Nucl. Phys. A 635, 231 (1998).

[50] D. A. Varshalovich, A. N. Moskalev, and V. K. Khersonskii, Quantum Theory of Angular Momentum (World Scientific, Singapore, 1988). 